\title{
A RAPIDLY SCANNING THREE-VELOCITY-COMPONENT
}

\section{LASER DOPPLER ANEMOMETER}

\author{
by
}

Marc M.E. Antoine

THESIS submitted to the Faculty of the

Virginia Polytechnic Institute and State University

in partial fulfillment of the requirements for the degree of Master of Science

in

Aerospace and Ocean Engineering

APPROVED :

R.L. Simpson, Chtirman

J.A. Schet?

B. Grossman

JUNE, 1985

Blacksburg, Virginia 


\title{
A RAPIDLY SCANNING THREE-VELOCITY-COMPONENT
}

\section{LASER DOPPLER ANEMOMETER}

\author{
by \\ Marc M.E. Antoine \\ R.L. Simpson, Chairman \\ Aerospace and Ocean Engineering
}

(ABSTRACT)

A rapidly scanning directionally-sensitive threevelocity - component laser Doppler anemometer has been developed. It permits scans through 3-dimensional flows to obtain space-time velocity information. Since it has lower signal-averaging bias than pointwise measurements, only short record times are required to obtain sufficient data for statistical averages and, hence, it enables rapid mapping of the $U, V$, and $W$ velocities in such flows.

A flexible optical system allows for easy variation of the fringe spacings as well as the location and the size of the measurement volume. The optical paths are equalized without use of any additional optical components. A Ronchi Ruling is employed to create a horizontal stationary fringe pattern, and a dual Bragg cell is used to produce sets of moving vertical fringes. It is also shown that a scheme proposed by other workers, relying on a fringe pattern moving in the on-axis direction, will not work. 
The Doppler frequency is independent of the position of the receiving optics, and only one photomultiplier tube is needed to receive the signals for all three velocity components. Measurements made on a stationary solid object as well as in a dispersion of particles in water show that signals with low noise level and high fringe visibility are obtained. 


\section{ACKNOWLEDGEMENTS}

The author wishes to express his deep appreciation to Dr. Simpson for his continuous encouragement and his constructive criticism during this research effort.

The author wishes to thank Dr. J. A. Schetz and Dr. B. Grossman for serving on the Advisory Committee.

Sincere appreciation is also extended to Dr. G. Indebetouw of the VPI Physics Department for his useful comments and suggestions.

The author would like to take this opportunity to thank his friends, in and outside of Blacksburg, who all contributed in their own way. 
TABLE OF CONTENTS

Introduction

Chapter I. Description of the Optical Configuration . . . . . . 5

Chapter II. The Fringe Pattern created by a Ronchi Ruling . . . 12 2.1 Diffraction . . . . . . . . . . . . . 12

2.2 Diffraction Phenomena in a Ronchi Ruling . . . 12 2.2.1 Influence of Geometry and Wavelength on the Diffraction Pattern of a Ronchi Ruling . . . . . . 13 2.2.2 Image formed by a Finite Number of Diffraction Orders

2.2.3 Periodic Variation of the Fringe Pattern . . 19 2.3 Fringe Pattern obtained with a Ronchi Ruling . 23 2.3.1 Fringe Visibility . . . . . . . . . . . . . 23 2.3.2 Experimental Verification of Ronchi Ruling Fringe Pattern . . . . . . . . . . . . . . . . 24 2.4 Optical Configuration for V-Component Measurements with a Ronchi Ruling . . . . . . . . . . . . 28 2.4.1 Influence of Lens Errors . . . . . . . . . . 28 2.4.2 Fo,cusing Lenses and Transmitting Lenses . . . 29

Chapter III. V-Component Measurements using a Ronchi Ruling . . 37 3.1 Instrumentation . . . . . . . . . . . . . . . . 37 
3.3.1 Scanner . . . . . . . . . . . . . . . 37

3.1.2 Receiving Optics . . . . . . . . . . . . 38

3.2 Horizontal Fringe Analysis and Velocity Bias

Considerations . . . . . . . . . . . . . . 43

3.3 Experimental Results . . . . . . . . . . . . 47

3.3.1 Measurements on Thin Stationary Wire . . . . 49

3.3.2 Measurements in Dispersion of Neutrally Buoyant Particles in Water . . . . . . . . . . . . 50

Chapter IV. Fringe Analysis for measuring $U$ - and W-

Components . . . . . . . . . . . . . . . . . 55

4.1 The dual Bragg Cell . . . . . . . . . . . . 56

4.2 Fringe Patterns for $\mathrm{U}$ - and W-Components . . . . 60

4.3 Doppler Frequencies for $U$ and $W$. . . . . . . 66

4.4 Equalizing Optical Path Lengths . . . . . . . 69

4.5 Laser Power Efficiency . . . . . . . . . . 70

Conclusions ..................... 72

References ...................... . 74

Vita ........................ 77 


\section{LIST OF ILLUSTRATIONS}

Figure 1. Top View of Optical Configuration of the System.

Figure 2. On-Axis View of 4 Beams forming the Measurement Volume.

Figure 3. Image of a Ronchi Ruling with 4 lines, using only 3 diffraction orders: $\mathrm{m}=-1,0,+1 . \quad 14$

Figure 4. Fraunhofer Diffraction in a Ronchi Ruling.

Figure 5. Influence of Number of Lines on Diffraction Pattern.

Figure 6. Fringe Pattern formed by a Finite Number of Diffraction Orders.

Figure 7. Variation of fringe pattern with number of diffraction orders:

Figure 8. Optical Configuration to study Ronchi Ruling. 26

Figure 9. Fringe patterns obtained with Ronchi Rulings. 27

Figure 10. Illustration of Lens Errors. . . . . . . . 30

Figure 11. Side View of Transmitting Lens Configuration. 35

Figure 12. View of Focusing Lenses (L3,L4), Ronchi Ruling and Concave Transmitting Lens (L1). . . . 36

Figure 13. View of Scanning Mirror. . . . . . . . . 39

Figure 14. Receiving Optics schematic diagram. . . . . 40

Figure 15. Ray Tracing for the Receiving Cylindrical Lenses, from Chehroudi (1983). . . . . . . 42

Figure 16. Number of Horizontal Fringes crossed by a Particle as Function of $\mathrm{V} / \mathrm{V}_{\mathrm{S}}$ and $\mathrm{U} / \mathrm{V}_{\mathrm{S}}$.

Figure 17. Influence of Particle Size on Doppler Signal Modulation.

Figure 18. Arrangement for V-component Measurements.

Figure 19. Doppler Signals for V-component. 
Figure 20. Frequency Shifting in Single Bragg cell. . 58

Figure 21. View of the Dual Bragg cell. . . . . . . . 59

Figure 22. Interference of Three Light Waves of Unequal Erequencies. . . . . . . . . . . . . 63

Figure 23. Fringe Patterns for $U$ and W. . . . . . 67 


\section{INTRODUCTION}

The Laser Doppler Velocimeter (LDV) has become a valuable tool for experimental investigations in fluid mechanics. The obvious advantage is its high frequency response and its ability to measure direction and magnitude of the velocity accurately and nonintrusively under conditions where other instruments provide questionable results or cannot be used at all.

Although commercial pointwise LDV systems are available, there is still a definite need for further instrument development. In order to obtain more detailed features in certain complicated flows, such as turbulent separated flows, "instantaneous" velocity profiles should be obtained. Therefore scanning LDV systems are needed because they yield nearly instantaneous velocity profiles and, concurrently, reduce the acquisition time of velocity information in at least one direction. Furthermore, three-dimensional measurements should be made so that important space-time flow-structural information can be obtained.

This work is concerned with the development and use of a rapidly scanning three-velocity-component laser Doppler anemometer. 


\section{Previous Work}

A brief description of few scanning LDA systems developed and used so far, is given by Chehroudi [1]. Bendick [9] described an on-axis scanning LDA that used a translational oscillating mirror. The operation of this design is limited to scan speeds of $0.4 \mathrm{~m} / \mathrm{s}$ due to the inertia of the moving optics.

A two-colour dual-beam backscatter LDA system accomplishing a scan by translating a lens in the direction of the optical axis, was reported by Grant and Orloff (1973). Scan rates were limited to $1.5 \mathrm{~m} / \mathrm{s}$ because of inertial considerations. More information concerning the application of this design is given by Orloff and Biggers [10] and orloff et al. [11] .

A backscatter scanning system was reported by Rhodes [12]. It is able to scan a distance of $30 \mathrm{~cm}$ at a frequency of $30 \mathrm{~Hz}$, and measures velocities at 16 discrete positions using a large rotating wheel containing 16 ports. For more information on this design, see Gartrell and Jordan [13] and Meyers [14].

An optical system capable of measuring true instantaneous velocity profiles was reported by Nakatani et al. [15]. Instead of using a moving scanning device it employed a cylindrical lens to form a vertical measurement volume along a straight line. The design is relatively impractical and expensive because a series of optical fibres connected to 
photodetectors is needed to collect data over a large scan range with good resolution.

The design of Durst et al. [16] uses a relatively large rotationally oscillating mirror in front of a conventional LDA optical system to scan the measurement volume perpendicular to the optical axis along an arc. Mean and RMS velocity profiles agreed well with pointwise measurements for low scan frequencies. The inertia of the oscillating mirror limited the scan frequency to about $15 \mathrm{~Hz}$.

Owen [17] developed a single velocity component scanning LDA and made measurements in both water and air flows. A six-sided rotating mirror was used and the scan rate was limited to $125 \mathrm{~Hz}$ due to restrictions on the data acquisition rates and the number of points required for one profile. Improvements were suggested for a second phase of the work which will enable two-component real-time scans through high speed air flows.

\section{Objective of this work}

Chehroudi and Simpson (1983) have developed a singlevelocity-component rapidly scanning LDA that can scan up to $150 \mathrm{~Hz}$ over distances of $40 \mathrm{~cm}$. This is the most rapid scan frequency reported to date. The proper operation of this design and its usefulness was demonstrated in a separated flow studied in a wind tunnel. Very good mean and RMS velocity profiles and profiles of the fraction that the flow 
moves downstream, are reported. Space-time correlations of recirculation flow regions were possible.

The objective of the present work is to modify and improve the optical system of Chehroudi and Simpson to measure three velocity components. 


\section{CHAPTER I. DESCRIPTION OF THE OPTICAL CONFIGURATION}

As shown in Figure 1, the incoming beam from an Argon ion laser goes through a pair of focusing lenses (L3,L4) and is incident onto a 500 lines per inch Ronchi Ruling (R). As discussed in Chapter 2, the Ronchi Ruling with horizontal lines produces a series of diffracted beams in a vertical plane due to Fraunhofer diffraction. These beams go through a transmitting lens combination $(L 1, L 2)$ and only the zeroth order $(m=0)$ and the two first-order diffracted beams $(m=+1$, -1) are retained, the higher orders being masked (S1). The first order diffracted beams are reflected (mirror M2) onto the scanning mirror (M5) and cross to form the measurement volume. The undiffracted beam (zeroth order) is reflected (mirrors M3, M4), and goes through a dual Bragg cell where it is split and frequency shifted. The outcoming unshifted beam is masked ( $\mathrm{S} 2$ ), whereas two of the shifted beams, one by +15 $\mathrm{MHz}$ and the other by $-25 \mathrm{MHz}$, are deflected by the scanning mirror onto a combination of vertical mirrors (M6, M7, M8, M9). These mirrors make the beams cross in the measurement volume (M.V.) at the desired angle. No additional optical components are necessary to equalize the optical path lengths in this arrangement.

In total, four beams cross to form the measurement volume: two first order diffracted beams from the Ronchi Ruling 


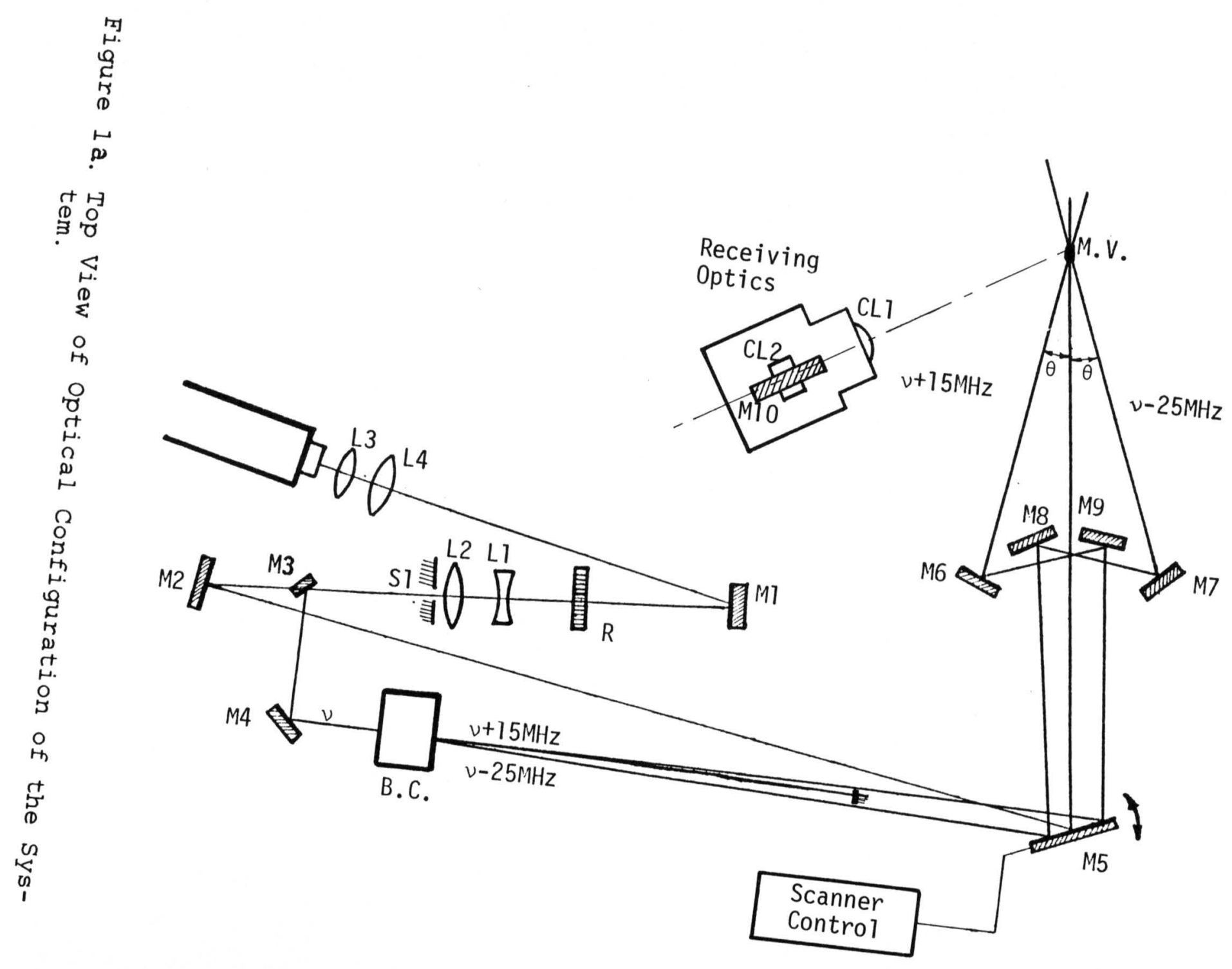


and two frequency shifted beams from the Bragg cell. This results in the formation of six fringe patterns (Fig.2), of which four are employed to make three-component velocity measurements.

A horizontal stationary fringe pattern is formed by the two first- order diffracted beams from the Ronchi Ruling, allowing the $V$-component to be measured by means of the vertical scanning motion. The frequency shifted beams from the Bragg cell cross with those diffracted beams from the Ronchi Ruling and produce moving fringe patterns at $15 \mathrm{MHz}, 25 \mathrm{MHz}$ and $40 \mathrm{MHz}$, due to optical beating. The latter yields a direct measurement of the U-component while the first two are fed into a frequency mixer and result in a signal around 10 $\mathrm{MHz}$ to give the $\mathrm{W}$-component.

The focusing lenses (L3,L4) determine the size of the measurement volume and optimize the fringe quality by ensuring that the beams cross at their waists. This is particularly important for obtaining parallel fringes inside the measurement volume. The transmitting lenses ( L1,L2) define the location of the measurement volume and allow the spacing of the horizontal fringe pattern to be varied freely. The choice of their characteristics is such that the lens errors are reduced. The other fringe spacings are determined by the angle $\theta$ and the wavelength of light. For a scan frequency of $54 \mathrm{~Hz}$, a scan range of $40 \mathrm{~cm}$ and a horizontal fringe 


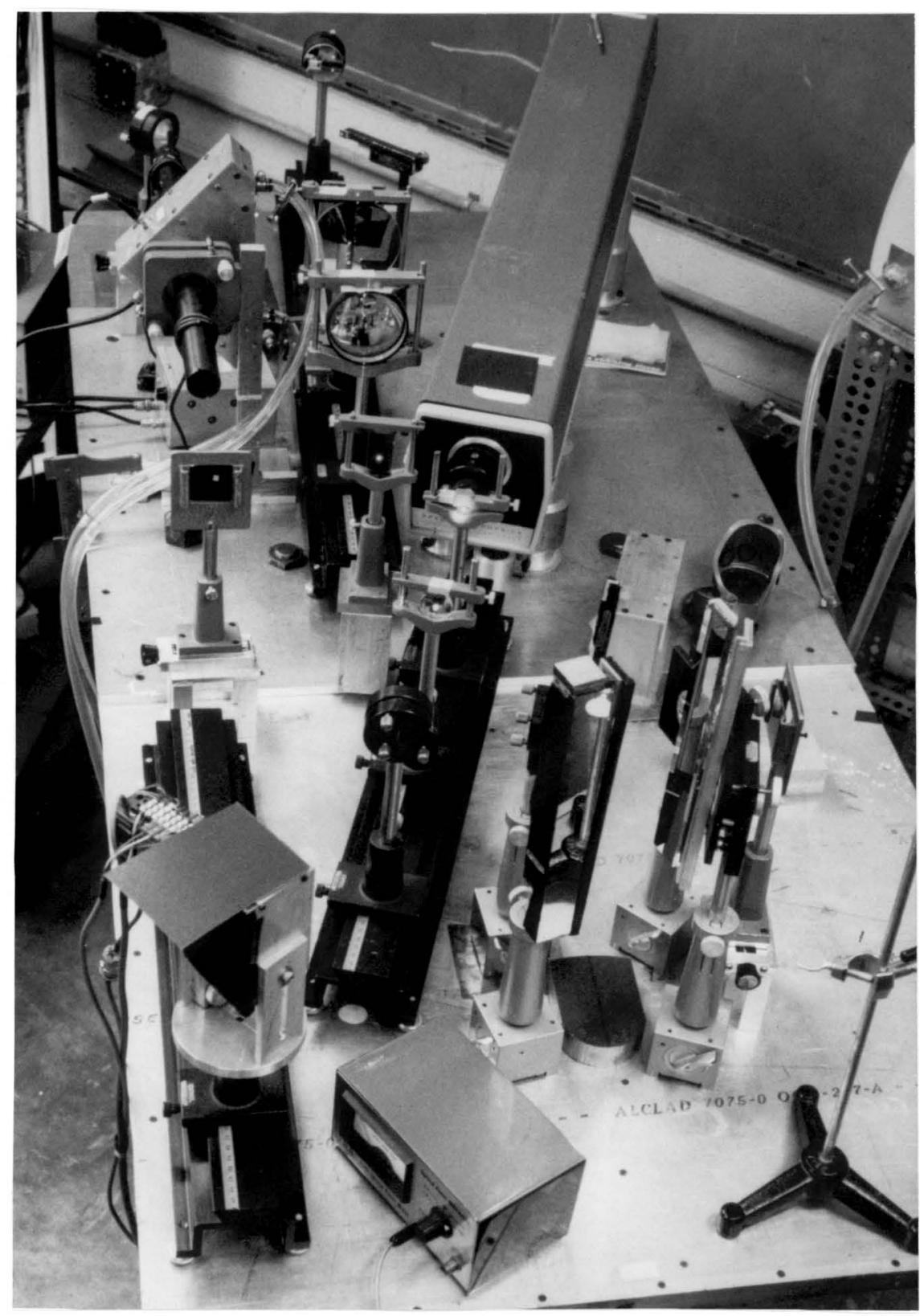

Figure 1b. View of the Optical Configuration. 


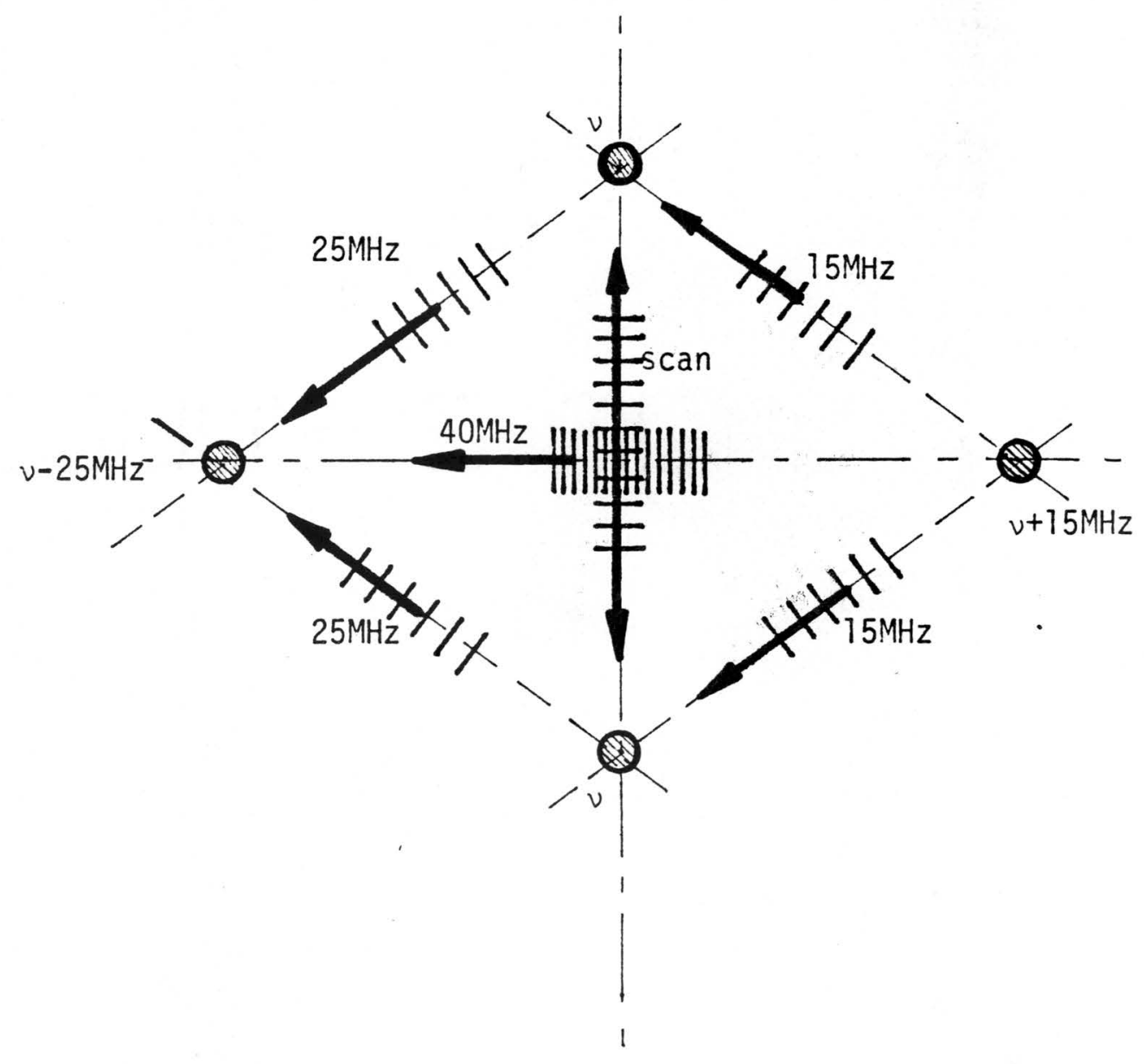

Eigure 2. On-axis View of the 4 Beams forming the Measurement Volume. 
spacing of $25 \mu \mathrm{m}$, the $\mathrm{V}$-component produces signals around 1 $\mathrm{MHz}$.

Hence, using only one PM-tube, signals around $1 \mathrm{MHz}, 10$ $\mathrm{MHz}$ and $40 \mathrm{MHz}$ are received and yield the V-, W-, and Uvelocity components respectively. Since a dual-beam configuration is employed (fringe-type LDA), the Doppler frequencies are independent of the position of the receiving optics. The Receiving Optics are configured in backscatter mode and consist of a combination of two cylindrical lenses (CL1,CL2), a long mirror (M10) and the photomultiplier tube. The cylindrical lenses and the mirror construct an image of the measurement volume on the PM-tube aperture for any position of scan. They are assembled inside a light enclosing frame and can move independently from each other in vertical and horizontal direction. 
Mirrors (front surface):

$\begin{array}{ll}\text { M1 ,M2,M4: } & \emptyset=43 \mathrm{~mm} \\ \text { M6,M7,M8, M9: } & 250 \times 32 \mathrm{~mm} \\ \text { M3: } & 10 \times 8 \mathrm{~mm} \\ \text { M70: } & 381 \times 51 \mathrm{~mm}\end{array}$

Scanning Mirror:

M5 :

$39 \times 26 \mathrm{~mm}$

Ronchi Ruling:

$\mathrm{R}:$

500 น/inch; $53 \times 24 \mathrm{~mm}$

Lenses:

L3:

double convex, $F L=38 \mathrm{~mm}$, $\theta=9 \mathrm{~mm}$;

L4: double convex, FL=119m, $\emptyset=26 \mathrm{~mm}$;

L2: double concave, $F L=-103 \mathrm{~mm}$, $\emptyset=87 \mathrm{~mm}$;

L1: $\quad$ plano convex, FL=254m, $\emptyset=101 \mathrm{~mm}$;

CL1: cylindrical, $F L=300 \mathrm{~mm}$, $60 \times 200 \mathrm{~mm}$;

CL2: cylindrical, $F L=86 \mathrm{~mm}$, $57 \times 60 \mathrm{~mm}$.

Bragg cell

B.C.:

transducers $\emptyset 25 \mathrm{~mm}$, frequencies $15.200 \mathrm{MHz}$, $24.550 \mathrm{MHz}$. 
CHAPTER II. THE FRINGE PATTERN CREATED BY A RONCHI RULING

\subsection{DIFFRACTION}

The term interference refers to the phenomenon that waves, depending on how they add together, intensify or weaken each other. There is no significant physical distinction between interference and diffraction but it has become customary to speak of interference when considering the superposition of only a few waves and diffraction when treating a large number of waves. Diffraction occurs as a wavefront of light passes by an obstruction and secondary wavefronts are generated and interfere. Such a diffraction pattern consists of regions of maximum and regions of minimum light intensity. These regions are called fringes.

\subsection{DIFFRACTION PHENOMENA IN A RONCHI RULING}

A Ronchi Ruling is a transparent plate ruled with black lines and clear, equal spaces. The Ruling used in the present work contains 500 lines per inch, which corresponds to a line spacing of $50 \mu \mathrm{m}$.

Aside from multiplicative factors, the Fraunhofer diffraction pattern is the Fourier transform of the electric field (i.e. the spatial distribution of amplitude and phase 
of the light waves) across a diffracting aperture [3], and can be obtained in the focal plane of a lens which is placed behind the aperture.

One of the most remarkable and useful properties of a converging lens is its inherent ability to perform twodimensional Eourier transformations. When a second lens is positioned behind the diffraction pattern, at a distance equal to its focal length, then an inverse Fourier transform of this pattern is taken and will result in the formation of an image of the diffracting aperture (Fig.3).

Since a Ronchi Ruling is used as a diffracting aperture, the image will consist of alternating dark and bright bands and, hence, a fringe pattern is obtained.

\subsubsection{INFLUENCE OF GEOMETRY AND WAVELENGTH ON THE} DIFFRACTION PATTERN OF A RONCHI RULING

A Ronchi Ruling can be described as a one-dimensional array consisting of $\mathrm{N}$ identical apertures of width $\mathrm{b}$ equally spaced by a distance a. Since it is an array of similar diffracting apertures its diffraction pattern can be represented by using the Array Theorem [4]. The intensity distribution of the Fraunhofer diffraction pattern is then found to be:

$$
I(\theta)=I_{0}(\sin N \alpha / \sin \alpha)^{2} \operatorname{sinc}^{2} \beta
$$




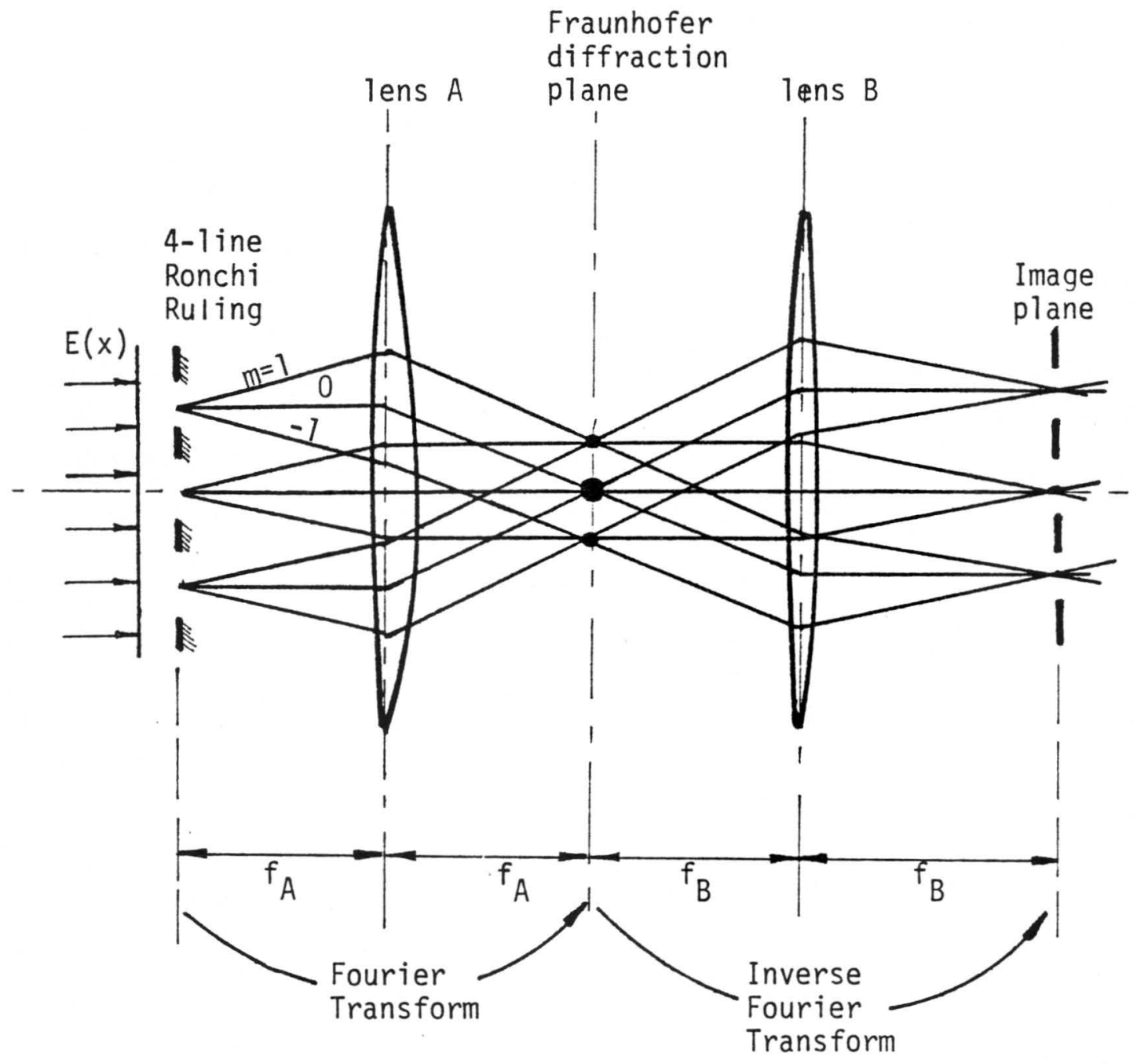

Eigure 3. Image of a Ronchi Ruling with 4 lines, using only 3 diffraction orders: $m=-1,0,+1$. 
where: $\theta=$ position angle in Eraunhofer plane

$$
\begin{aligned}
I_{0} & =I(0) / N^{2} \\
\alpha & =k / 2 a \sin \theta \\
\beta & =k / 2 b \sin \theta \\
k & =2 \pi / \lambda
\end{aligned}
$$

This intensity distribution is represented in Fig.4a. Each slit on the Ruling would generate an intensity distribution given by $\operatorname{sinc}^{2} \beta$. Superimposed, the various contributions yield a multiple wave interference system modulated by the single diffraction envelope $\operatorname{sinc}^{2} \beta$.

In summary, the modulation is determined by the line width $(b / \lambda)$, and the peaks within the envelope are determined by the line spacing $(a / \lambda)$. From (1) we can readily deduce the influence of geometry of the Ruling and the wavelength on the diffraction pattern, as illustrated in Fig. 4b:

- increasing the line width (b) makes the envelope more narrow but leaves the spacing between peaks, which depends on $a / \lambda$ only, unchanged;

- increasing the line spacing (a) moves the peaks closer together, but the envelope remains the same;

- increasing the wavelength $(\lambda)$ makes the envelope 
broader and moves the peaks further apart, but the number of peaks within the envelope, which depends on $\mathrm{a} / \mathrm{b}$ only, does not change.

The intensity distribution of the diffraction pattern for a Ronchi Ruling (i.e. $a=2 b$ ) and the influence of the number of lines is illustrated in Fig.5. . An increase in the number of lines augments the maxima and makes them sharper. Simultaneously, the subsidiary maxima decrease and become of negligible intensity.

\subsubsection{IMAGE FORMED BY A FINITE NUMBER OF DIFFRACTION ORDERS}

If all diffraction orders are used (infinitely many) then the resulting image would be identical to the diffracting aperture. Using only a finite number of diffraction orders is equivalent to truncating the Fourier series to a finite number of terms and therefore the image is not an exact duplicate of the Ronchi Ruling. An expression for the distribution in the image plane can readily be developed since it is the inverse Fourier transform of the diffraction pattern. Applying the Array Theorem to the Ronchi Ruling, the amplitude distribution $E_{R}(x)$, assuming unit incident amplitude, can be written (for $N+1$ lines) as:

$$
E_{R}(x)=\sum_{n=-N / 2}^{N / 2} E(x) * \delta(x-n \cdot a)
$$




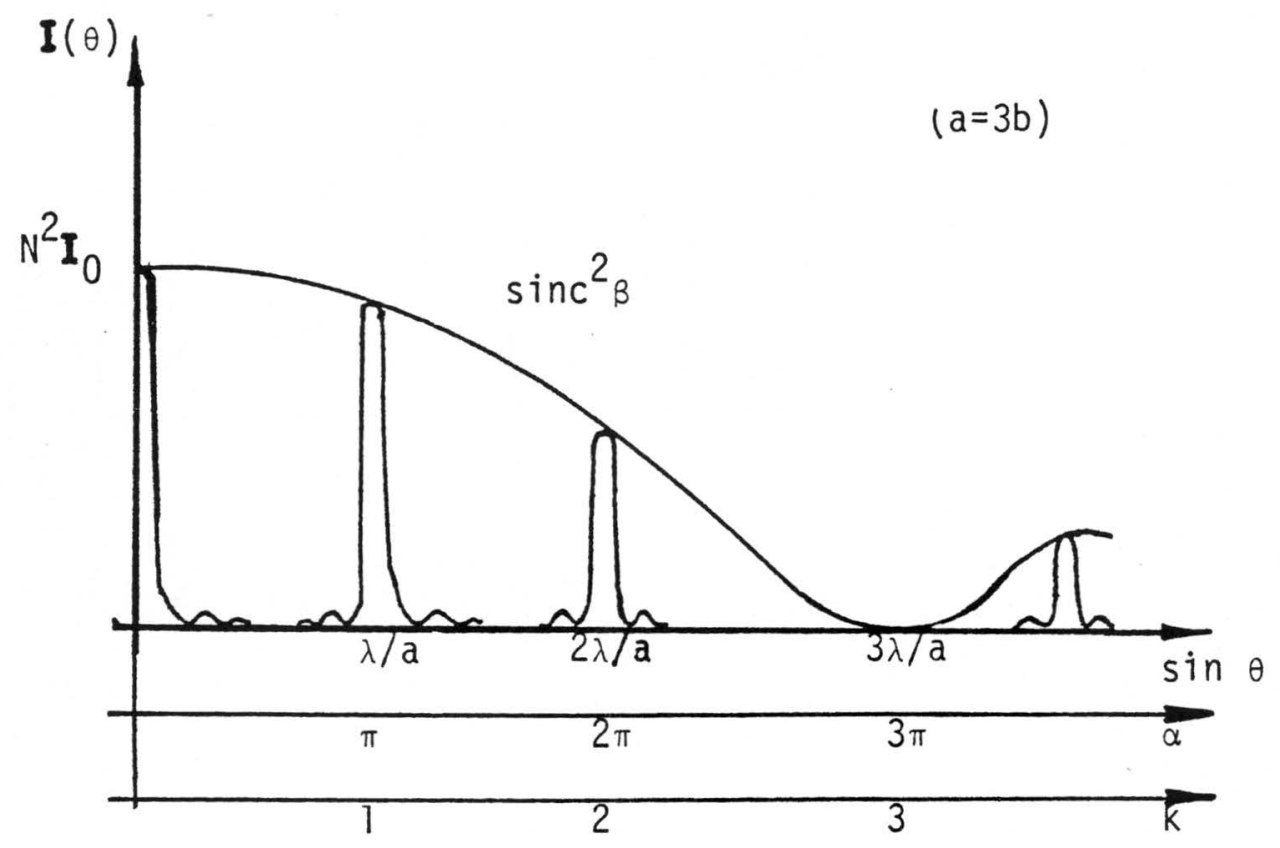

$4 a$.

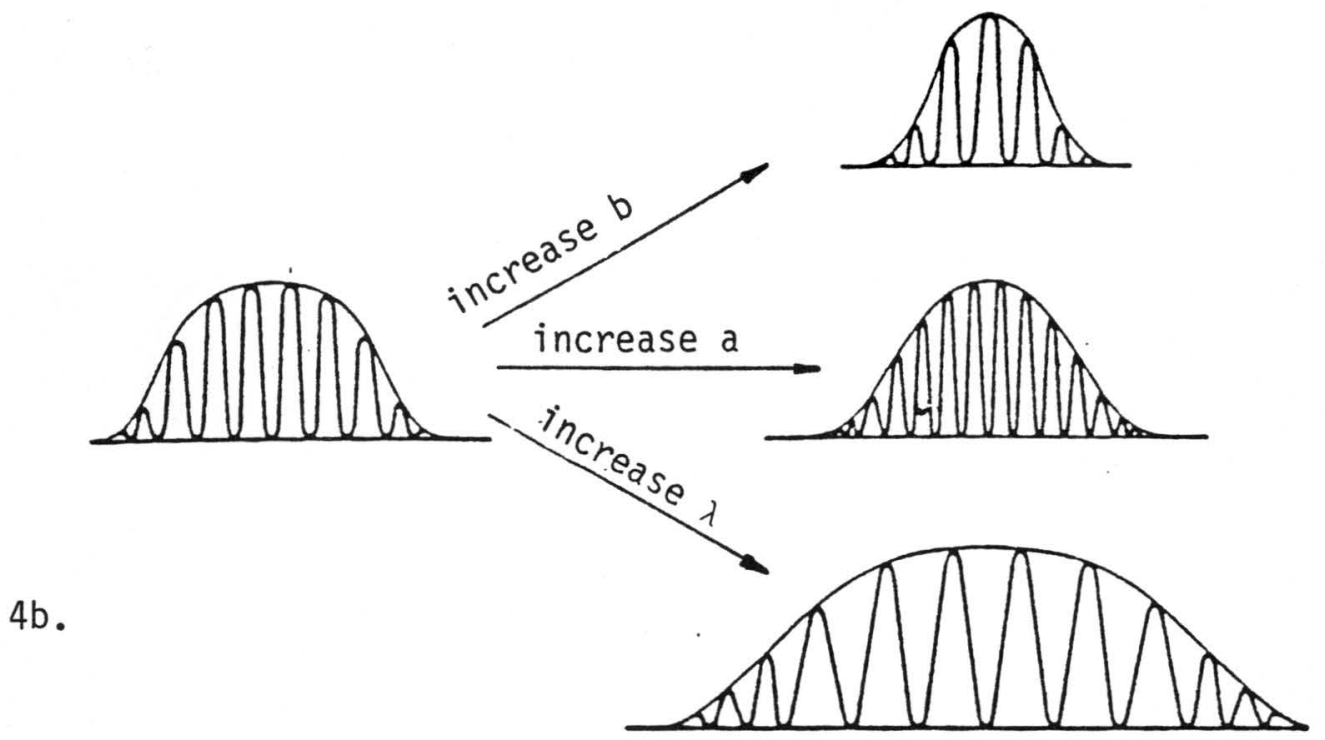

Figure 4. Eraunhofer Diffraction in a Ronchi Ruling. 
where: $\quad E_{R}(x)=$ amplitude distribution across Ronchi Ruling

$$
\begin{aligned}
& \begin{aligned}
E(x)= & \text { amplitude distribution across one slit } \\
= & 1 \text { for }|x|<b / 2 \\
& \quad 0 \text { for }|x|>b / 2
\end{aligned} \\
& \delta(x)=\text { Dirac delta function } \\
& \text { * denotes convolution. }
\end{aligned}
$$

Then the Fraunhofer diffraction pattern is obtained by taking the Fourier transform (denoted by ):

$$
\tilde{E}_{R}(\xi)=\sum \tilde{E}(\xi) \cdot \delta(\xi-n / a),
$$

and if only $\mathrm{m}+1$ diffraction orders are retained to generate the image, by taking the inverse Fourier transform, the distribution in the image plane, $E_{i}(x)$, becomes:

$$
\begin{aligned}
E_{i}(x)= & \left.\sum_{n=-m / 2}^{m / 2} E^{-1} \tilde{E}(\xi) \cdot \delta(\xi-n / a)\right\} \\
& =\sum_{n=-m / 2}^{m} \tilde{E}(n / a) \exp (-j x n / a \sin \theta)
\end{aligned}
$$

$$
\text { where: } \quad \begin{aligned}
\tilde{E}(\xi) & =b \operatorname{sinc}(\pi b \xi) \\
& =b \operatorname{sinc} \beta
\end{aligned}
$$


This process is illustrated in Fig. 6.

\subsubsection{PERIODIC VARIATION OF THE FRINGE PATTERN}

A significant difference in the fringe pattern occurs depending on the number of diffraction orders that are retained. Consider all diffraction orders in the Fraunhofer plane being masked, except for two (e.g. $m=+1$ and $m=-1$ ). Then the waves will be in phase all along the bisector of their propagation direction, and interfere constructively (Fig.7a). Hence, the fringe pattern will remain unchanged along the optical axis (z-axis) and will merely be translated perpendicularly to this axis. Moreover, it can easily be shown from equation (2) that the intensity distribution is sinusoidal in this case.

If more than two diffraction orders are retained (e.g. $\mathrm{m}=+1,0,-1)$ then these waves will be in phase along the optical axis only if their optical path lengths differ by a multiple of the wavelength. Hence, the fringe pattern will change periodically along the optical axis, from locations where the waves interfere constructively, yielding distinct fringes, to locations where they interact destructively (no fringes). (Fig.7b) 


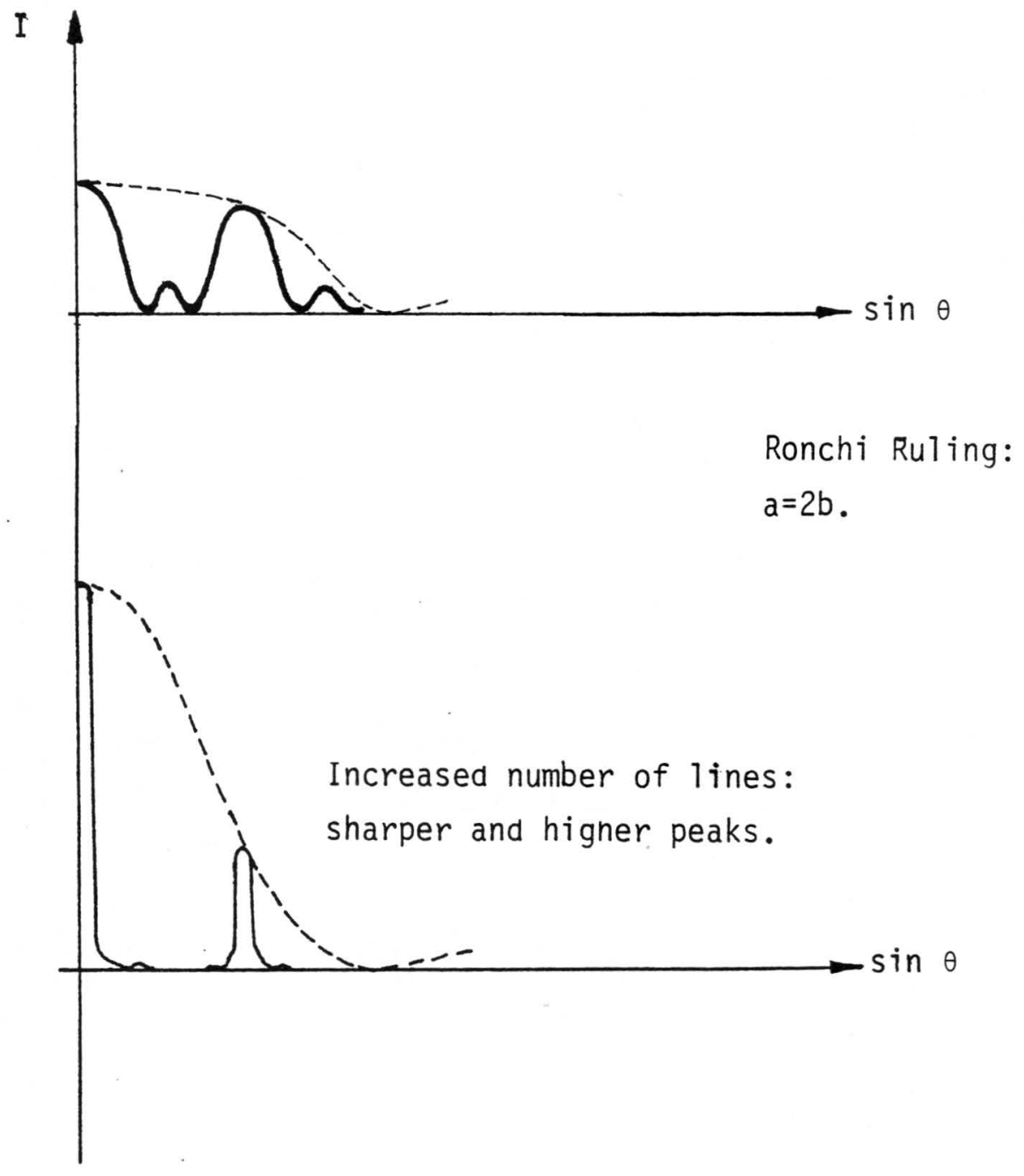

Figure 5. Influence of Number of Lines on Diffraction Pattern. 


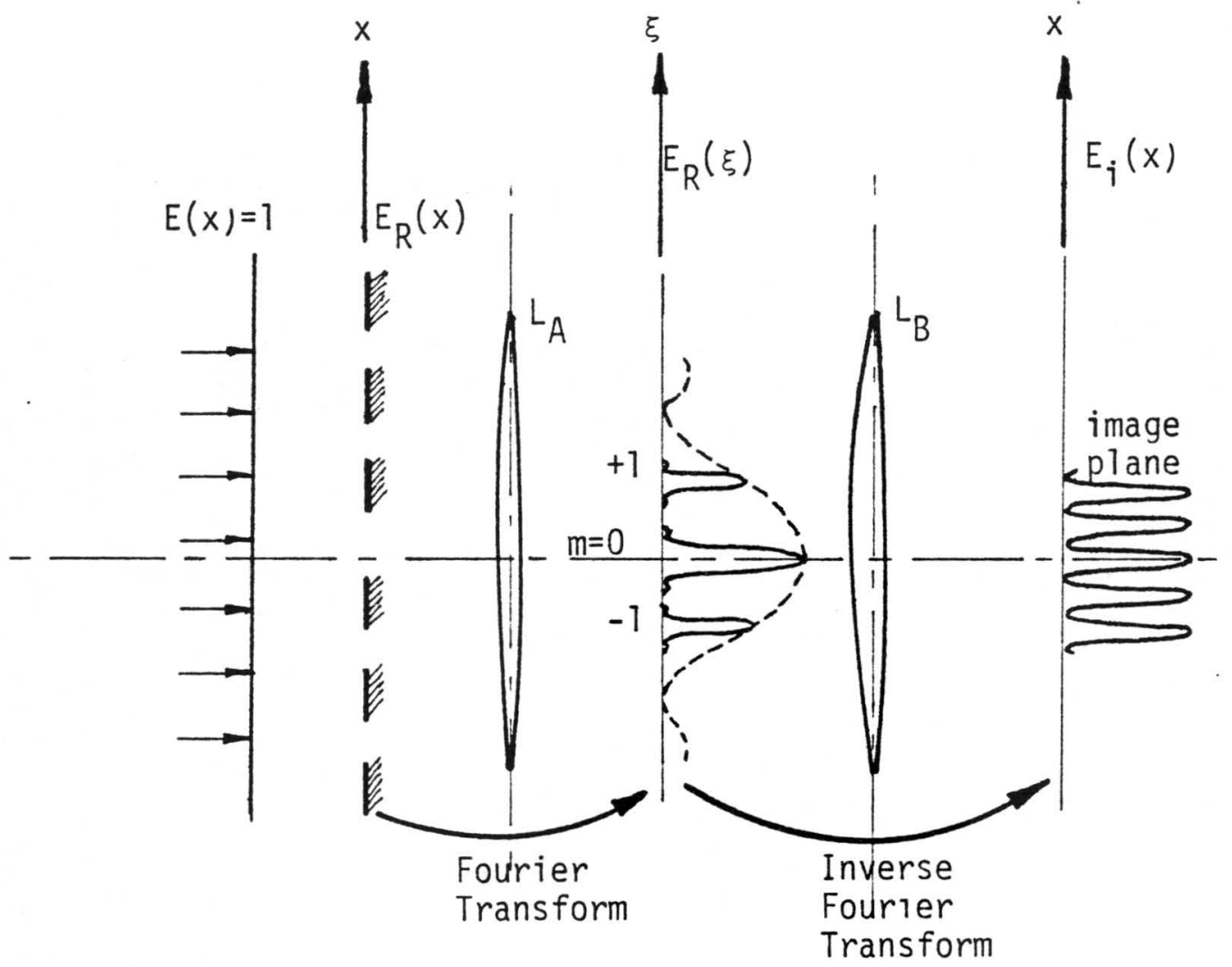

Eigure 6. Eringe Pattern formed by a Finite Number of Diffraction Orders. 

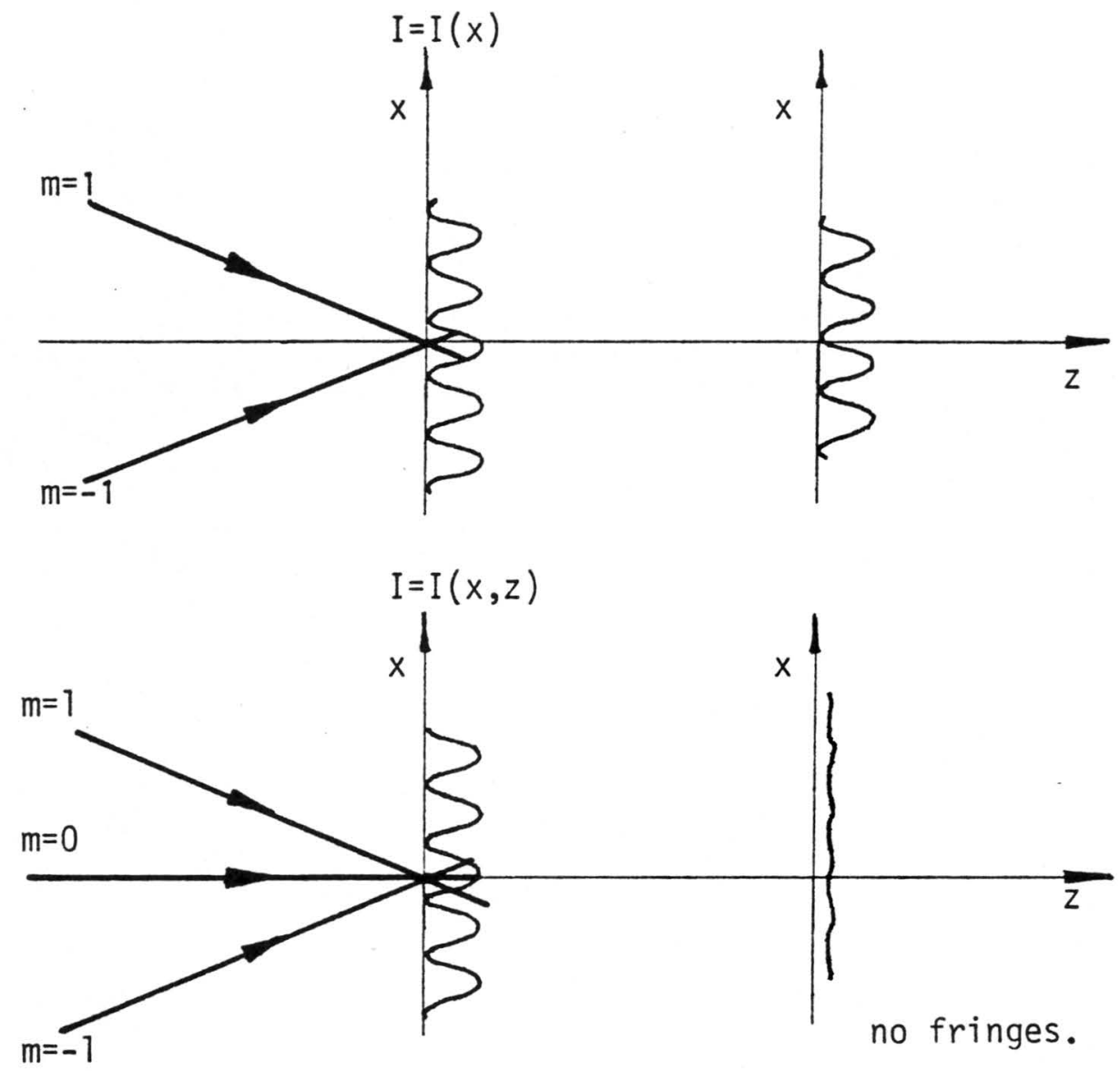

Eigure 7. Variation of fringe pattern with number of diffraction orders:

(a) two diffraction orders $(m=1,-1)$

(b) three diffraction orders $(m=1,0,-1)$. 
For this case it can easily be shown that the intensity distribution is not sinusoidal anymore but contains higher harmonics.

\section{Choice of Diffraction Orders}

The above considerations are particularly important in obtaining a horizontal fringe pattern to make V-velocity measurements with the Ronchi Ruling. Since we need a fringe pattern which remains unchanged in space, we cannot use more than two diffraction orders. Moreover, to guarantee high fringe visibility as well as equal, equidistant fringes, only two symmetric diffraction orders can be used. They have equal intensity and do not introduce higher harmonics in the fringe pattern. Therefore, in order to obtain maximum laser power in the measurement volume, the two first-order diffracted beams $(m=-1,+1)$ are employed to produce the horizontal fringes.

\subsection{FRINGE PATTERN OBTAINED WITH A RONCHI RULING}

\subsubsection{FRINGE VISIBILITY}

An important parameter in interference phenomena is the fringe visibility (FV). It is the measure of the fringe contrast and is of great importance for the quality of the 
Doppler signals in LDV, since it affects the SNR greatly. It can be defined as:

$$
F V=\left(I_{\max }-I_{\min }\right) /\left(I_{\max }-I_{\min }\right)
$$

where: $\quad I_{\max }=\operatorname{maximum}$ fringe intensity

$$
I_{\min }=\text { minimum fringe intensity, }
$$

and, hence, must have a value between zero and unity.

\subsubsection{EXPERIMENTAL VERIFICATION OF RONCHI RULING FRINGE PATTERN}

Instead of moving particles that scatter the light waves, a relative motion between fringe pattern and detector was introduced, with a scanner, such that an intensity variation results on the detector surface. This is achieved by adjusting the fringe spacing and the photodetector aperture so that the fringes are carried across the aperture by the scanner.

As shown in Figure 8, a beam from a $5 . \mathrm{mW}$ He-Ne laser goes through a beam expander (BXI) before being incident onto the Ronchi Ruling ( $R$ ). A finite number of diffraction orders, formed with a lens (LI), are retained by using an iris (D2) and deflected by a scanning mirror (M1). These 
diffracted beams are recombined with a lens (L) and focused onto the slit of the PM-tube by means of an objective (BX2). Figure 9 clearly illustrates the phenomena described above, using a 100 lines per inch Ronchi Ruling. Signals with fringe visibility of $95 \%$ are obtained. The envelope is Gaussian due to the Gaussian intensity distribution of the laser beam itself, as shown in Figure 9a. The fringe pattern produced with only two identical diffraction orders results in a sinusoidal intensity distribution (Fig.9b), whereas more diffraction orders generate higher harmonics, as shown in Figure 9c. When using the zeroth and first order diffracted beams $(m=-1,0,1)$, the fringe pattern changes periodically along the optical axis, and destructive interference is present. In that case the fringe visibility will tend to zero, as shown in Figure 9d. 


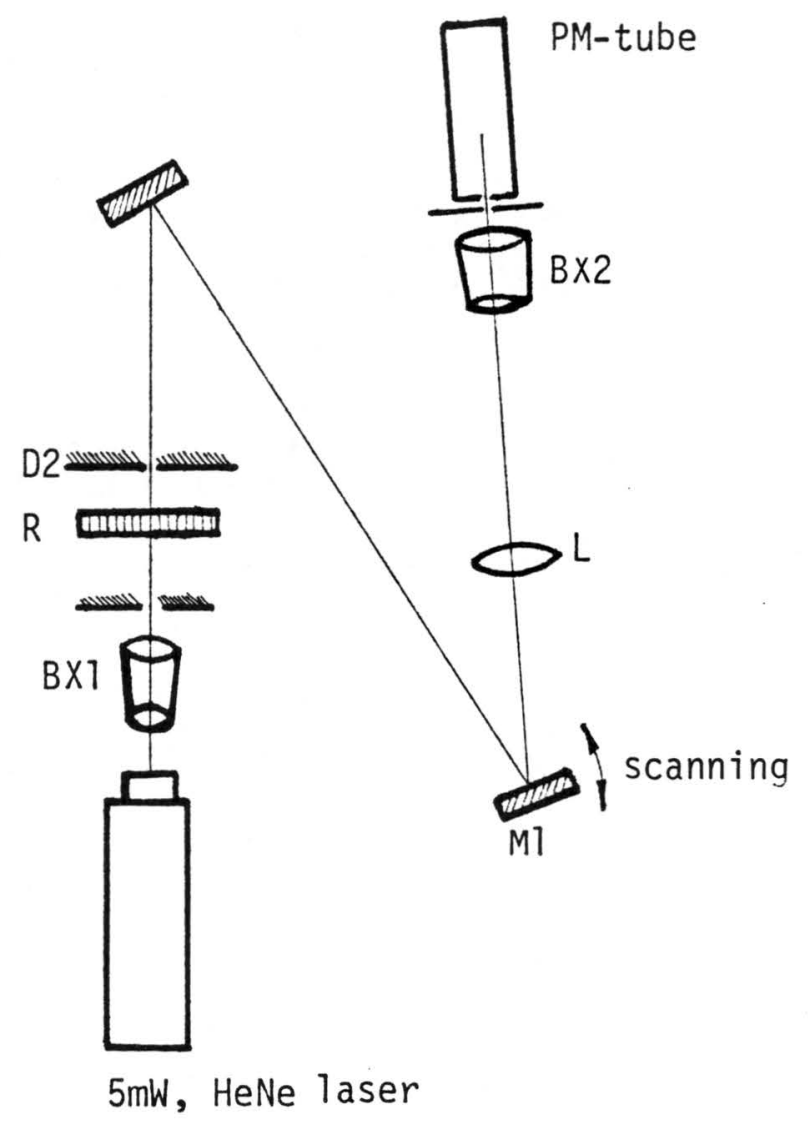

Figure 8. Optical Configuration to study Ronchi Ruling. 


\section{(a)}

Fringe visibility $=95 \%$

(b)

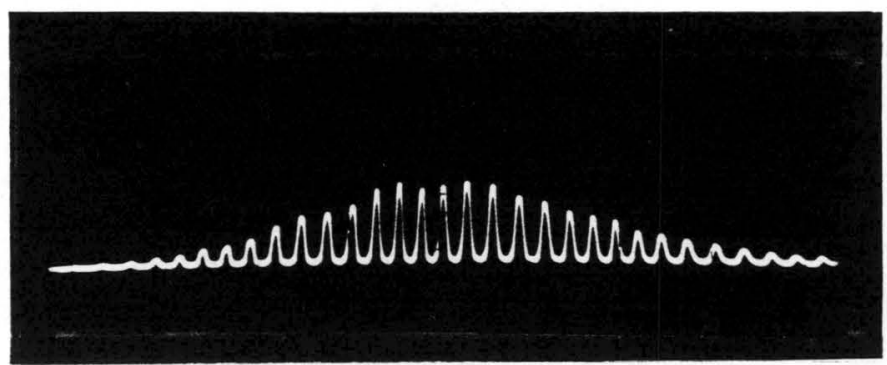

Sinusoidal distribution using diffraction orders $\mathrm{m}=-1,+1$

(c)

Higner harmonics when using more than two diffraction orders
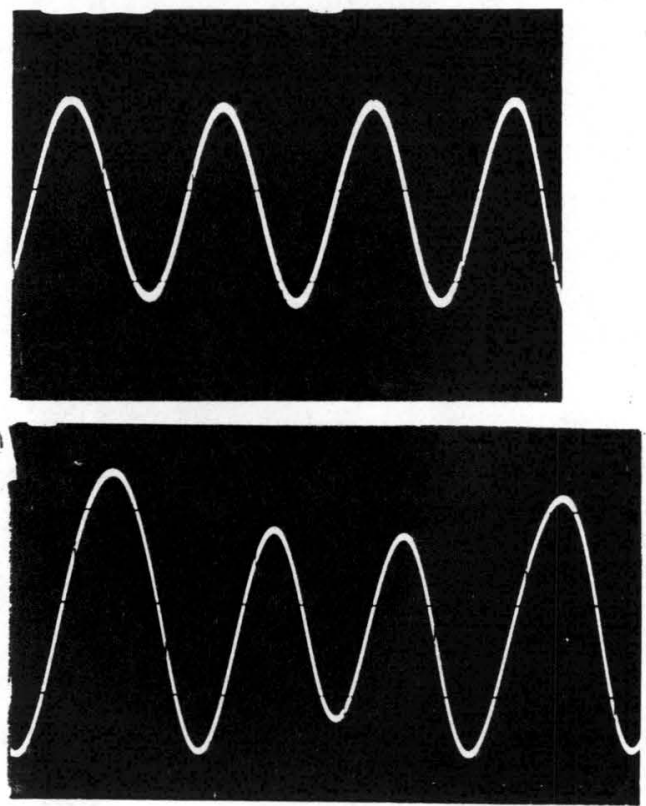

(d)

In case (c) the fringe visibility can tend to zero along the optical axis

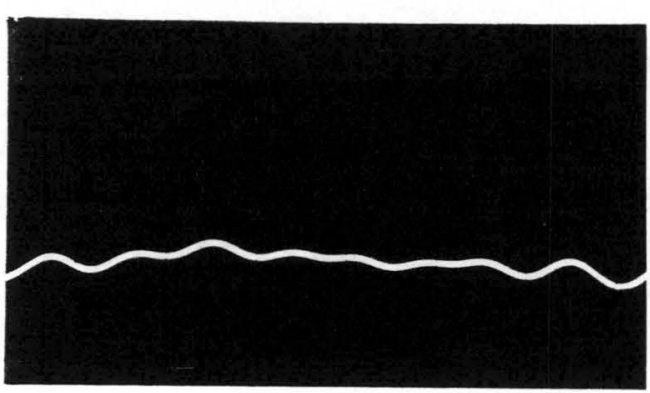

Figure 9. Fringe patterns obtained with Ronchi Rulings. 


\subsection{OPTICAL CONFIGURATION FOR V-COMPONENT MEASUREMENTS}

WITH A RONCHI RULING

\subsubsection{INFLUENCE OF LENS ERRORS}

The lens imperfection that led to the most difficulties is spherical aberration. In terms of geometrical optics it means that rays incident on the lens parallel to its axis cross the axis at different points, and that the focal point of a beam depends on the distance, $h$, of that incident beam to the lens axis. (Fig.10a)

This causes errors in the measured Doppler frequencies when beams do not cross at their waists, since the measurement volume then contains non-parallel fringes (Fig. 10b). Particles Pl crossing the measurement volume nearer the lens will result in a higher frequency than particles P2 with the same velocity crossing at the other end.

Another error that occured was due to diffraction at the focus of a lens. Due to the wave nature of light there exists a deviation from the strict straight line propagation assumed in geometrical optics. This means that even a perfect lens cannot bring a parallel beam to a true point of focus, and therefore the beam waist was located nearer the lens than the focal plane (F). (Fig. 10c) 
The deviation was greater the smaller the diameter of the incident parallel beam.

Therefore appropriate steps were taken to reduce these lens errors and ensure an optimum beam intersection. This was achieved by using two particular lens combinations: the focusing lenses and the transmitting lenses.

\subsubsection{FOCUSING LENSES AND TRANSMITTING LENSES}

These two lens combinations are used in order for the beams to cross at their waists, and to form a measurement volume of desired fringe spacing at the desired location in space.

The focusing lenses are positioned between the laser and the Ronchi Ruling and consist of a pair of double convex lenses L3 and L4. (Fig.1) They allow the location of the beam waists to be varied freely and, hence, determine the size of the measurement volume. Therefore they also influence the fringe visibility and the laser power density in the measurement volume.

The transmitting lenses produce the measurement volume by forming an image of the Ronchi Ruling. They determine the fringe spacing and quality, as well as the location of the measurement volume. The configuration which was first tried consisted of one double convex lens. The magnification of the image, $M$, is given by: 


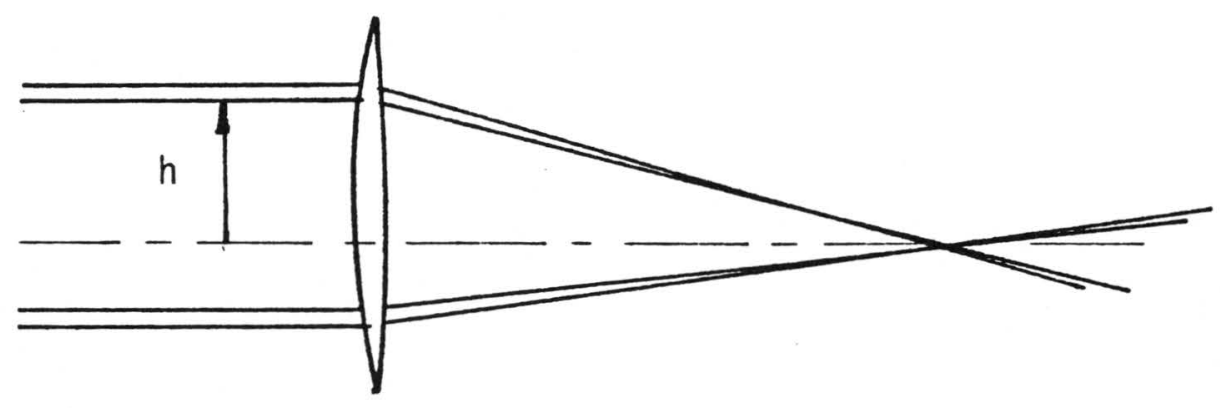

(a) Spherical Aberration

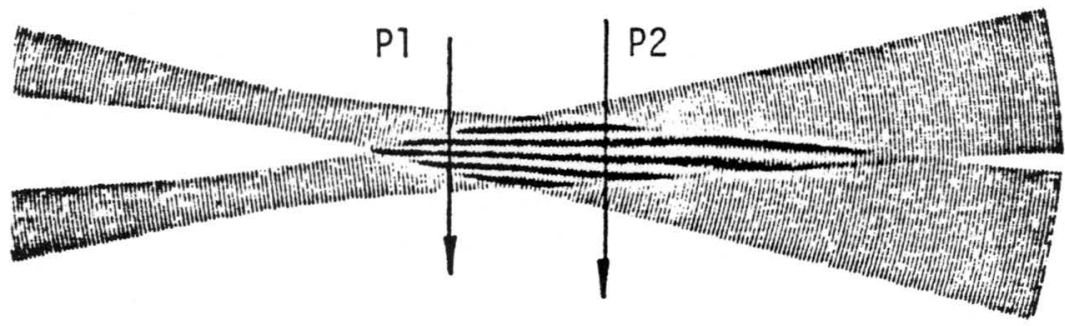

(b) Non-parallel fringes when beams do not cross at waists

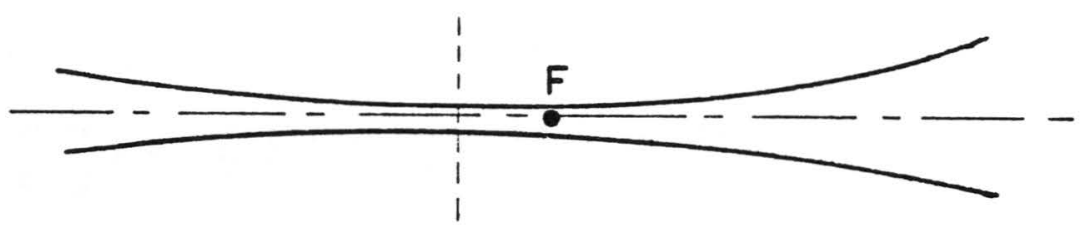

(c) Diffraction at focus of lens

Figure 10. Illustration of Lens Errors. 


$$
M=s_{i} / s_{0}
$$

where: $s_{0}=$ distance from object to lens

$$
s_{i}=\text { distance from image to lens, }
$$

and where $s_{0}$ and $s_{i}$ are related to the focal length, $f$, through the thin lens formula:

$$
1 / f=1 / s_{0}+1 / s_{i}
$$

There were several restrictions to this configuration. To obtain both large image and magnification factor less than one, the object distance has to be greater than the image distance:

$$
\begin{aligned}
& s_{\circ}, s_{i} \text { large } \\
& s_{0}>s_{i}
\end{aligned}
$$

This implies that the focal length of the lens has to be large and, hence, the overall dimensions of the optical configuration become very large. Moreover, the lens error cannot be reduced in this set-up.

Therefore another configuration was used (Fig.1), consisting of a combination of a double-concave and a planoconvex lens, L1 and L2.. This arrangement offers several advantages. The beams cross at their true waist by making 
them divergent with a concave lens in front of the planoconvex lens. The error due to spherical aberration is reduced since a plano-convex lens shows substantially less spherical aberration than a double-convex lens. The fringe spacing, determined by the magnification factor of this lens combination, and the location of the measurement volume can be varied easily without the overall dimensions of the optical system becoming large. This can be shown by considering the design criteria and equations for the transmitting lenses L1 and L2.

\section{Design Criteria}

(a) The distance between the last lens (L2) and the measurement volume should be at least $225 \mathrm{~cm}$.

(b) The horizontal fringe spacing should equal $25 \mu \mathrm{m}$.

(c) The fringe visibility should be maximized.

\section{Design Equations}

Eor a combination of two thin lenses, L1 and L2, separated by a distance $d$, the image distance is given by [4]:

$$
s_{i 2}=\frac{f_{1} d-f_{2} s_{01} f_{1}\left(s_{01}-f_{1}\right)}{d-f_{2}-s_{01} f_{1} /\left(s_{01}-f_{1}\right)}
$$

where: $f_{1}$ focal length of first lens 


$$
\begin{aligned}
& f_{2}=\text { focal length of second lens } \\
& s_{\circ 1}=\text { distance from object to first lens } \\
& s_{i 2}=\text { distance from last lens to image }
\end{aligned}
$$

The magnification of the image is given by the product of the individual magnifications of the lenses, and becomes:

$$
M_{F}=\frac{f_{1} s_{i 2}}{d\left(s_{01}-f_{1}\right)-s_{01} f_{1}}
$$

\section{Design Values}

The image formation of the Ronchi Ruling through the lens pair L1,L2 will yield the desired fringe pattern. It involves 6 parameters $\left(f_{1}, f_{2}, d, s_{O 1}, s_{i 2}, M_{F}\right)$ and is described by 2 equations $(2,3)$. The design criteria specify the parameters $\mathrm{s}_{i 2}$ and $\mathrm{M}_{\mathrm{F}}$ :

from (a): $\quad s_{i 2}=225 \mathrm{~cm}$

from (b): $\quad M_{E}=0.5$, since the Ronchi Ruling has a line spacing of $50 \mu \mathrm{m}$ and the fringe spacing should equal $25 \mu \mathrm{m}$.

Using lenses with focal lengths $f_{1}=-10.3 \mathrm{~cm}, f_{2}=25.4 \mathrm{~cm}$ and solving equations (2) and (3) yields: 


$$
\begin{aligned}
& \mathrm{s}_{01}=150 \mathrm{~cm} \\
& \mathrm{~d}=19 \mathrm{~cm}
\end{aligned}
$$

The additional advantage of this optical configuration is its flexibility in adjusting the fringe spacing as well as the size and location of the measurement volume. This is particularly useful in correcting for discrepancies that occur between the actual values and the design values. These differences are due to the thin lens approximations used in the calculated values

$$
\text { e.g. measured: } \begin{aligned}
\mathrm{s}_{\circ 1} & =110 \mathrm{~cm} \\
\mathrm{~d} & =19 \mathrm{~cm} \\
\mathrm{~s}_{i 2} & =340 \mathrm{~cm} \\
\lambda_{\mathrm{EV}} & =25 \mu \mathrm{m}
\end{aligned}
$$

$$
\text { calculated: } \quad \begin{aligned}
s_{\mathrm{ol}} & =110 \mathrm{~cm} \\
\mathrm{~d} & =19 \mathrm{~cm} \\
\mathrm{~s}_{\mathrm{i} 2} & =239 \mathrm{~cm} \\
\lambda_{\mathrm{FV}} & =36 \mu \mathrm{m}
\end{aligned}
$$




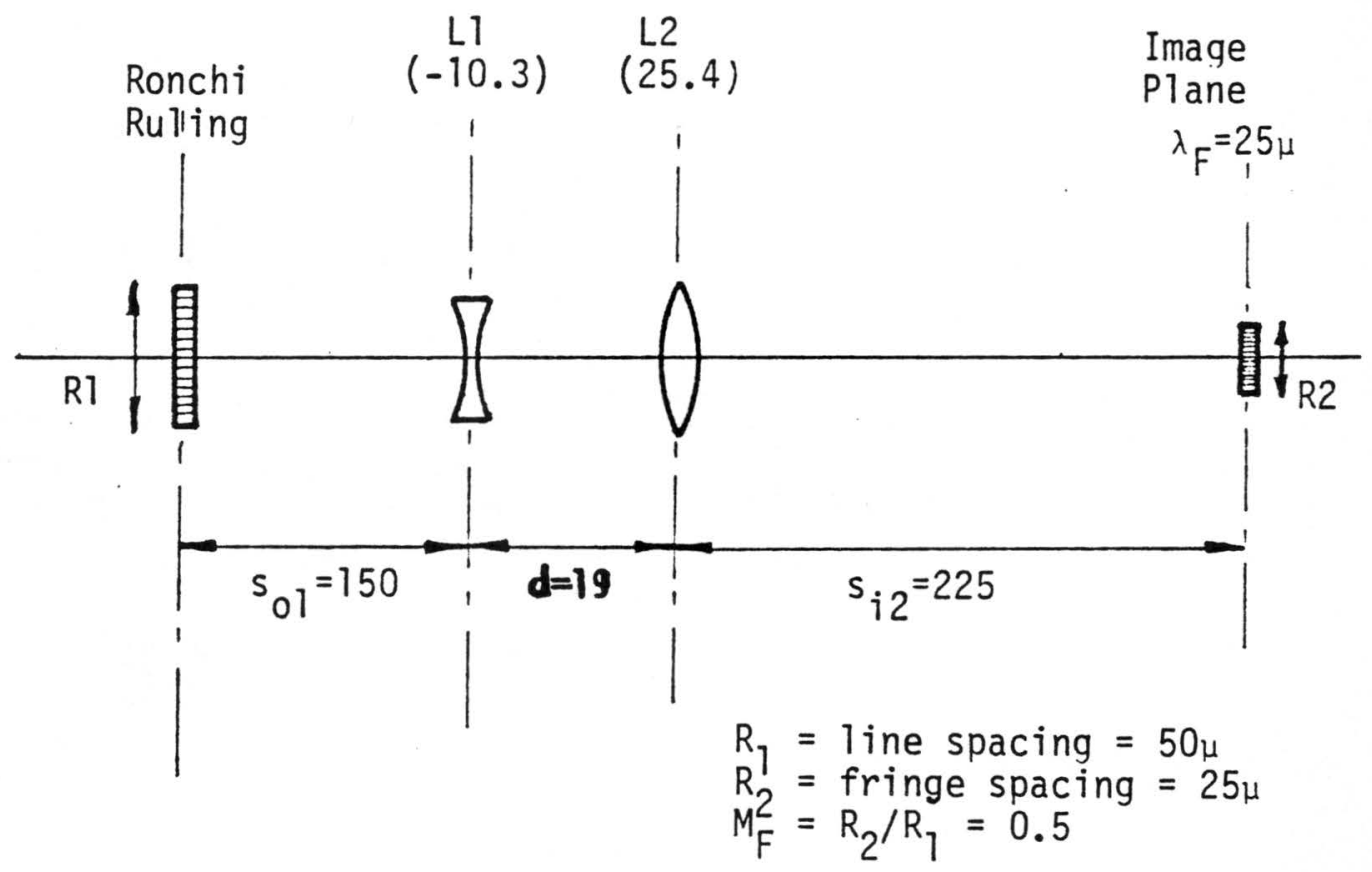

(distances in $\mathrm{cm}$ )

Figure 11. Side View of Transmitting Lens Configuration. 


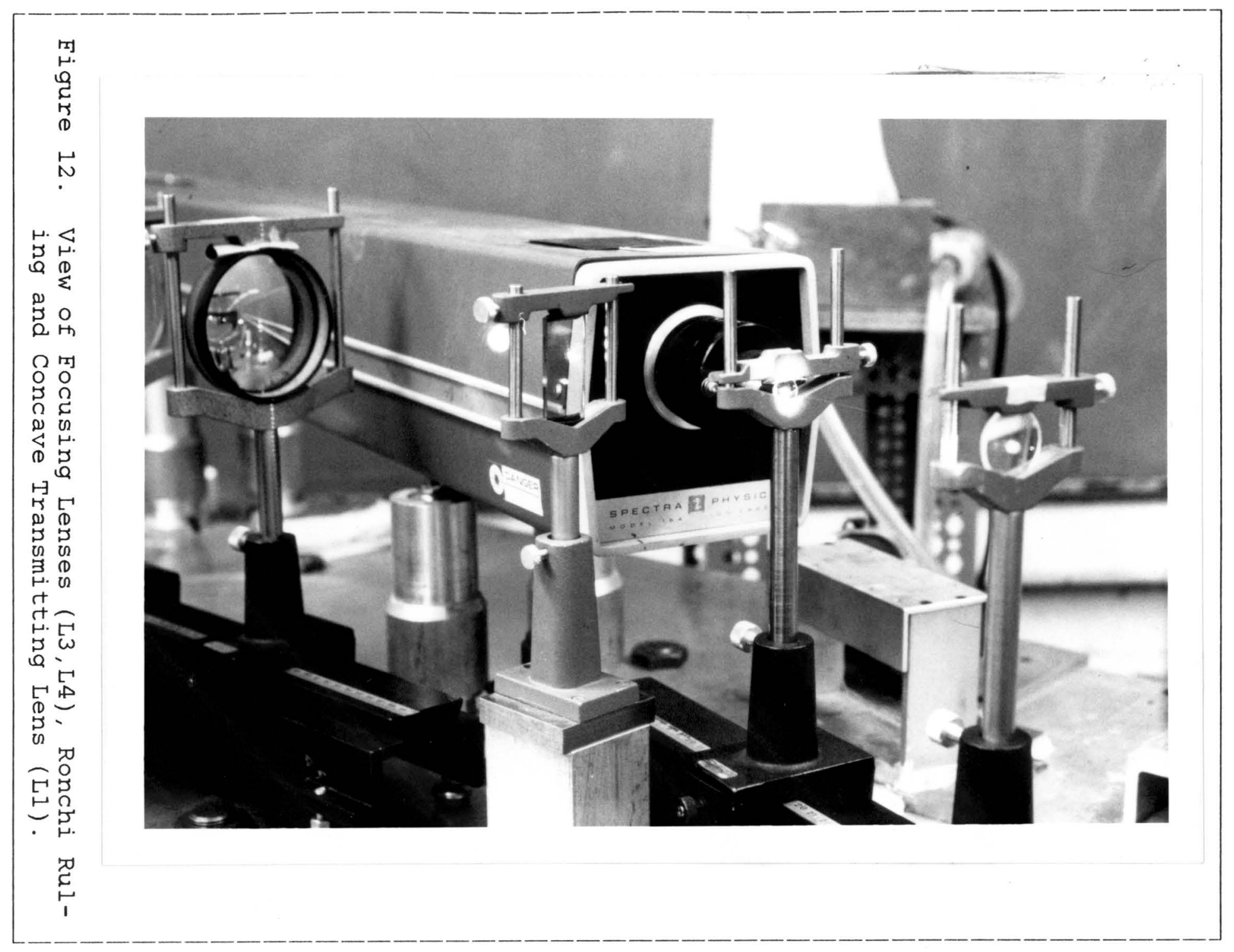


CHAPTER III. V-COMPONENT MEASUREMENTS USING A RONCHI RULING

\subsection{INSTRUMENTATION}

\subsubsection{SCANNER}

The scanner serves two purposes. It performes rapid scans in the vertical direction, thereby allowing nearly instantaneous velocity profiles to be obtained. Concurrently, it introduces a relative motion of the horizontal fringes with respect to a stationary scatterer and, hence, permits directional discrimination of the V-velocity.

The G300PDT scanner from General Scanning Inc. is a moving iron galvanometer with a position transducer designed specifically for closed-loop operation. This transducer operates by detection of capacitance variation between the rotating armature and a set of stationary electrodes. The controller includes a heater control regulating the temperature of the scanner. The mirror is a front surface mirror with Alsio coating, and is flat to one wavelength per $\mathrm{cm}$. The shaft wobble is typically below 5 arc-seconds and the signal response time is $10 \mathrm{~ns}$. Chehroudi tested the linearity of the sawtooth generator which drives the scanner [1]. It was found that this voltage was linearly proportional to 
the angular position of the mirror, and consequently to the measurement volume position, with a correlation coefficient of .999956.

\subsubsection{RECEIVING OPTICS}

The receiving optics consist of two cylindrical lenses (CL1,CL2), a long front surface mirror (M10), and the PM-tube, all assembled inside a black box having a side door for any adjustment. (Fig.14)

The first cylindrical lens (CLI) gathers light and enlarges the image of the measurement volume in the streamwise direction. The mirror deflects the light onto the second cylindrical lens (CL2) which constructs the image of the measurement volume on the PM-tube aperture for any positions of scan. Both lenses and mirror can be moved independently from each other in vertical and horizontal direction. The receiving optics were positioned in backscatter mode for the present work.

Figure 15 shows the ray tracing for the cylindrical lenses. CL2, working in a vertical plane, and CL1, working in a horizontal plane, can both be described by the thin lens equations :

for CL2: $\quad 1 /\left(Z+X_{1}\right)+1 / X_{2}^{\prime}=1 / E_{2}$ 


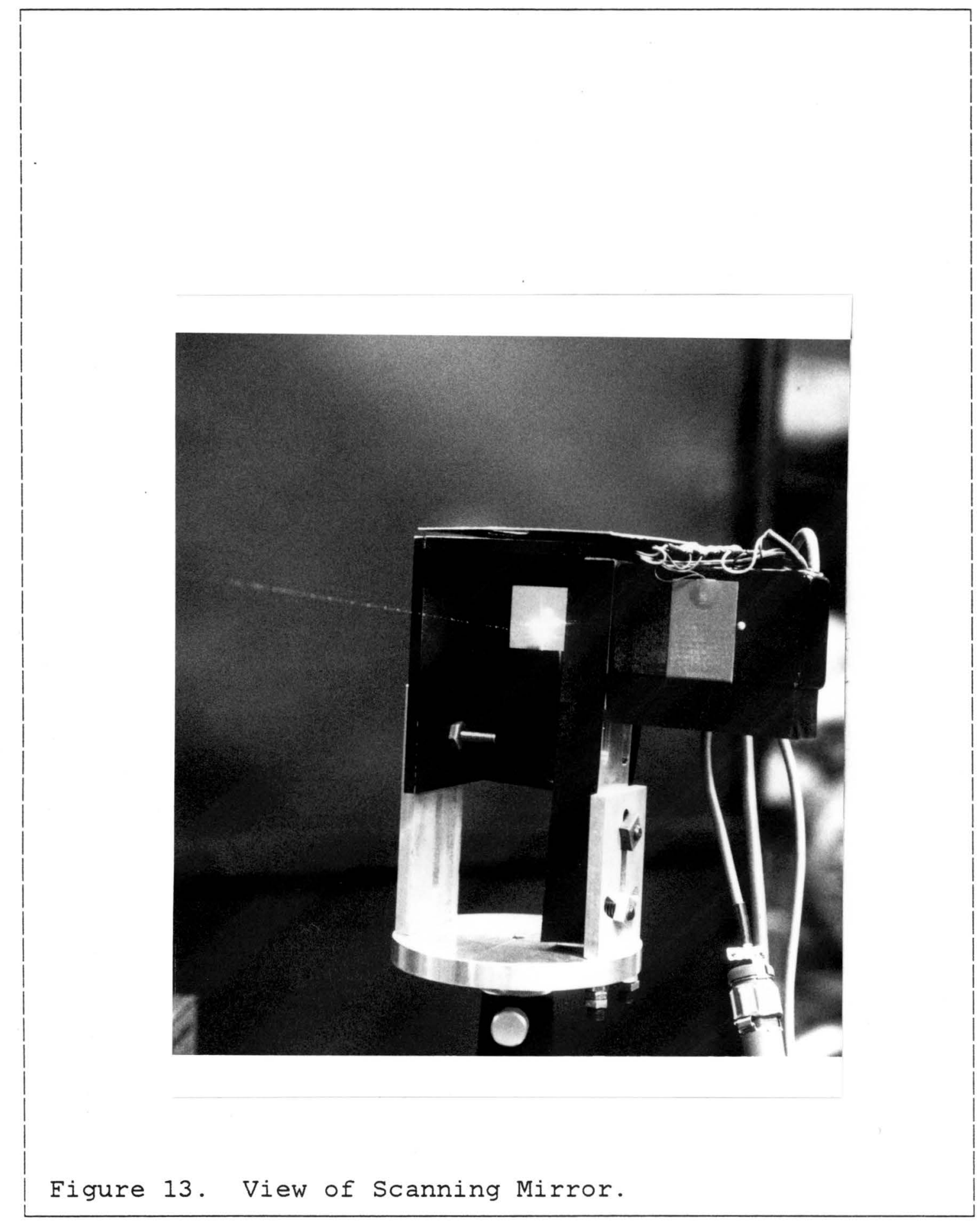




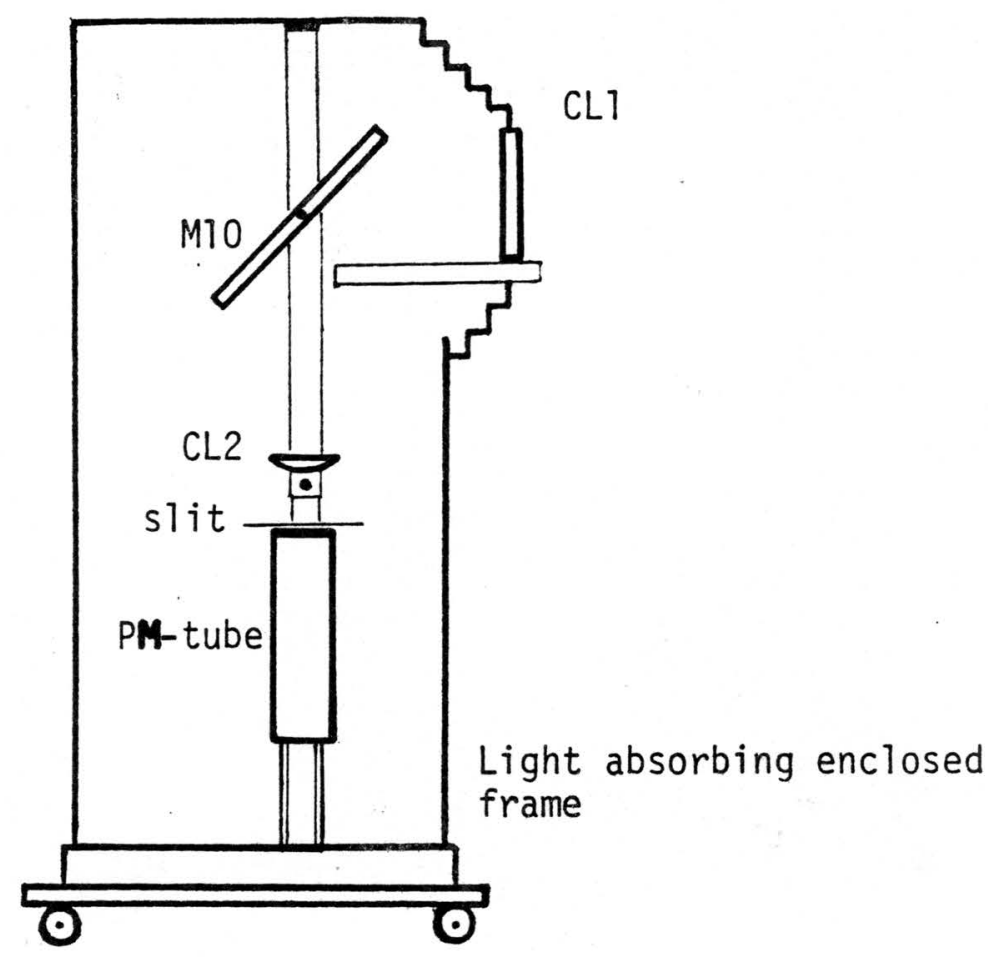

Figure 14. Receiving Optics schematic diagram. 


$$
o_{1} / I_{f}=\left(z+x_{1}\right) / x_{2}^{\prime}
$$

for $\mathrm{CL} 1: \quad 1 / \mathrm{X}_{1}+1 / \mathrm{X}_{1}^{\prime}=1 / \mathrm{F}_{1}$

where: $\mathrm{Z}=$ distance between CL1 and CL2

$$
\begin{aligned}
& \mathrm{X}_{1}=\text { distance from measurement volume to CL1 } \\
& \mathrm{x}_{2}^{\prime}=\text { distance from CL2 to PM-tube } \\
& \mathrm{X}_{1}^{\prime}=\text { distance from CL1 to PM-tube } \\
& \mathrm{O}_{1}=\text { scan range } \\
& \mathrm{I}_{\mathrm{f}}=\text { image of scan on PM-tube slit } \\
& \mathrm{F}_{1}=\text { focal length of CL1 } \\
& \mathrm{F}_{2}=\text { focal length of CL2 }
\end{aligned}
$$

A design procedure based on these equations is described by Chehroudi [1]. For $F_{1}=300 \mathrm{~mm}, O_{1}=381 \mathrm{~mm}$ and $I_{f}=30 \mathrm{~mm}$, one could determine $\mathrm{F}_{2}$ to equal $86 \mathrm{~mm}$ for $\mathrm{X}_{1}=485 \mathrm{~mm}$. 


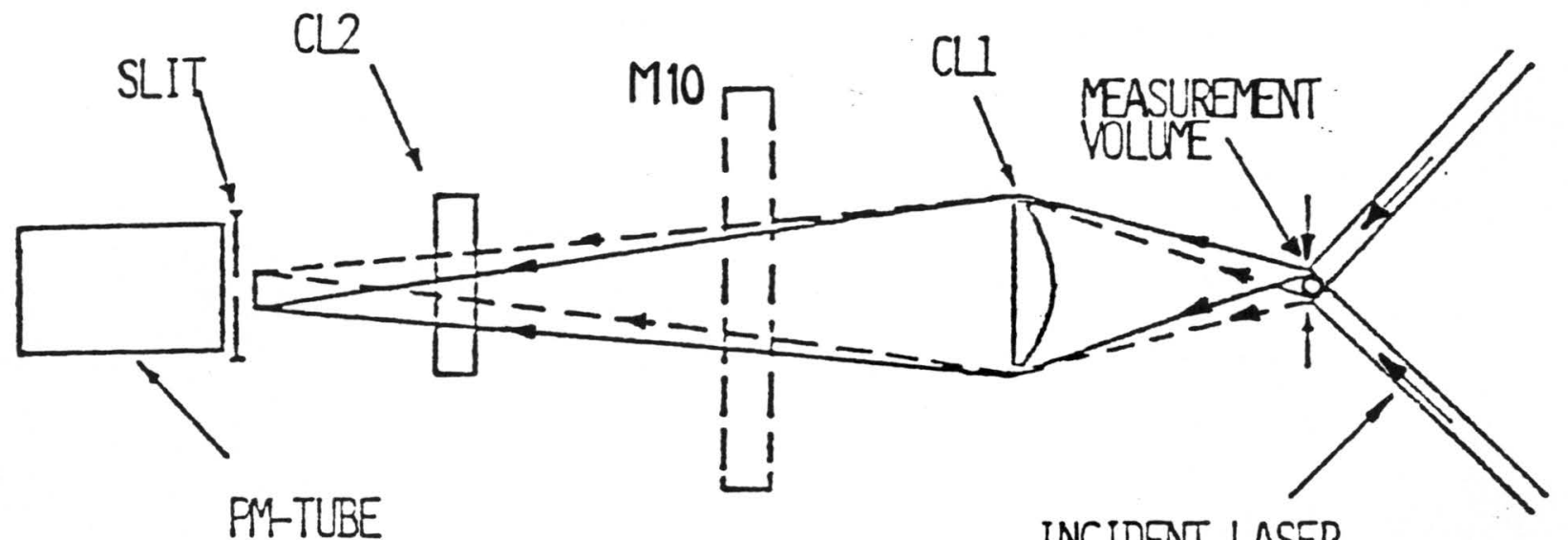

INCIDENT LASER BEAM

(a) TOP VIEN

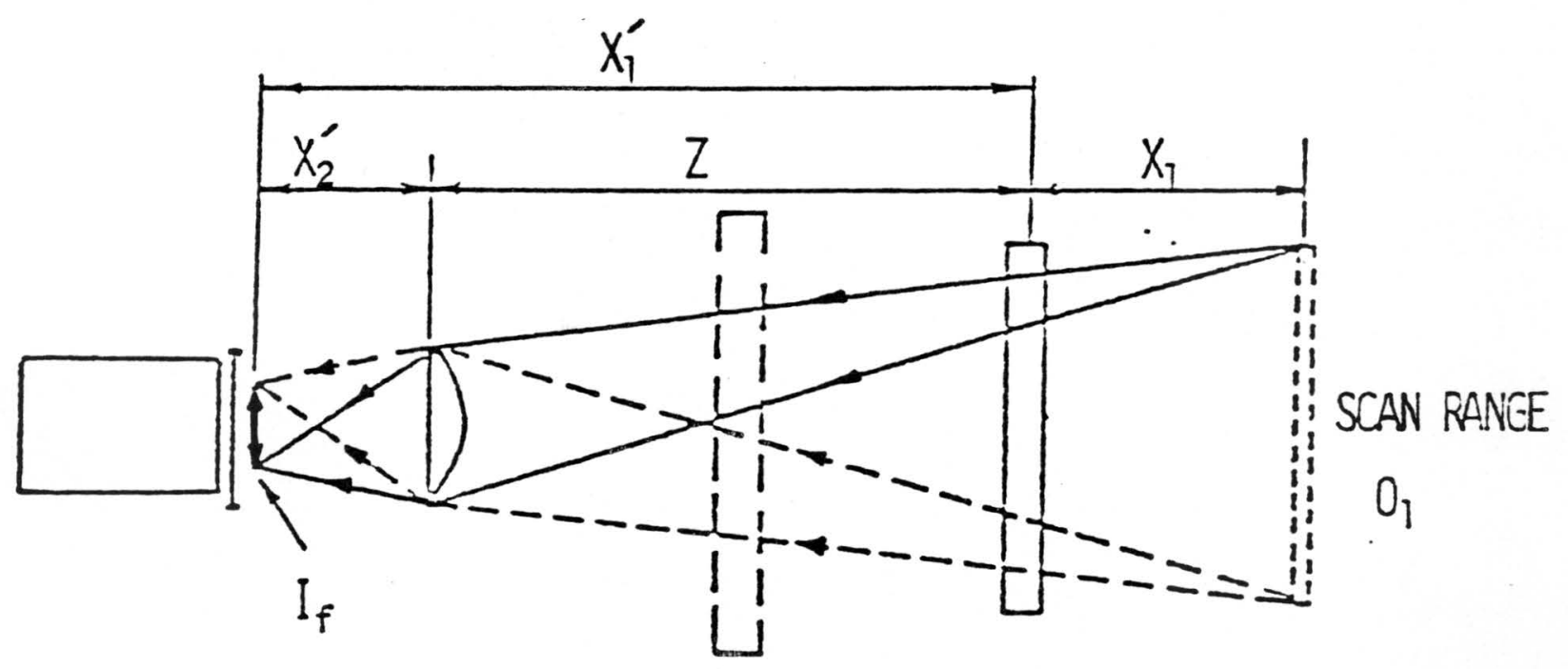

(b) SIDE VIEN

Figure 15. Ray Tracing for the Receiving Cylindrical Lenses, from Chehroudi (1983). 


\subsection{HORIZONTAL FRINGE ANALYSIS AND VELOCITY BIAS CONSIDERATIONS}

Chehroudi [1] derived an equation to represent the number of fringes crossed by a particle entering the measurement volume. The analysis is based on the assumption that the measurement volume is two-dimensional and circular in shape, and the particle motion is dominated by its streamwise velocity. From a practical point of view, the maximum number of fringes crossed is of interest, and it was shown to decrease as the scan speed increased. Using a counter type processor which needs 32 cycles per signal burst would limit the scan speed to approximately $200 \mathrm{~m} / \mathrm{s}$ to insure that there were enough vertical fringes crossed. This upper limit for the scan speed was much greater than the one obtainable with the oscillating mirror. In the present design a dual Bragg cell is used, which results in a higher fringe velocity in the $\mathrm{U}$-direction (at $40 \mathrm{MHz}$ ). This allows much higher scan speeds.

A similar analysis can be performed for the horizontal fringes. The time for a particle to travel one horizontal fringe is given by:

$$
t_{V}=\lambda_{F v} /\left(v-V_{S}\right)
$$

where: $\quad \lambda_{F V}=$ horizontal fringe spacing 


$$
\begin{aligned}
& \mathrm{V}=\text { vertical particle velocity component } \\
& \mathrm{V}_{\mathbf{S}}=\text { scan velocity (vertical) }
\end{aligned}
$$

As indicated in Figure 16a, the residence time of a particle in the measurement volume is given by:

$$
\begin{aligned}
t_{\text {res }} & =A B / V_{r} \\
& =d \cos (\theta+\phi-\pi) /\left\{U^{2}+\left(V_{s}-V\right)^{2}\right\} 1 / 2
\end{aligned}
$$

where: $\quad V_{r}=$ relative velocity of particle with respect to measurement volume

$\mathrm{d}=$ diameter of measurement volume

$\mathrm{U}=$ horizontal particle velocity component.

Setting the cosine term in eqn.(2) equal to 1, which physically means that the particle passes through the center of the measurement volume, we can obtain an expression for the maximum number of horizontal fringes crossed:

$$
\left(\mathrm{N}_{\mathrm{v}}\right)_{\max }=\left(t_{\text {res }}\right)_{\max } / t_{\mathrm{v}}
$$

and write it in a non-dimensional form as:

$$
\lambda_{\mathrm{EV}} \mathrm{N}_{\mathrm{v}_{\max }} / \mathrm{d}=\left|\mathrm{V} / \mathrm{V}_{\mathrm{s}}-1\right| /\left\{\left(\mathrm{U} / \mathrm{V}_{\mathrm{S}}\right)^{2}+\left(1-\mathrm{V} / \mathrm{V}_{\mathrm{S}}\right)^{2}\right\}^{1 / 2}
$$




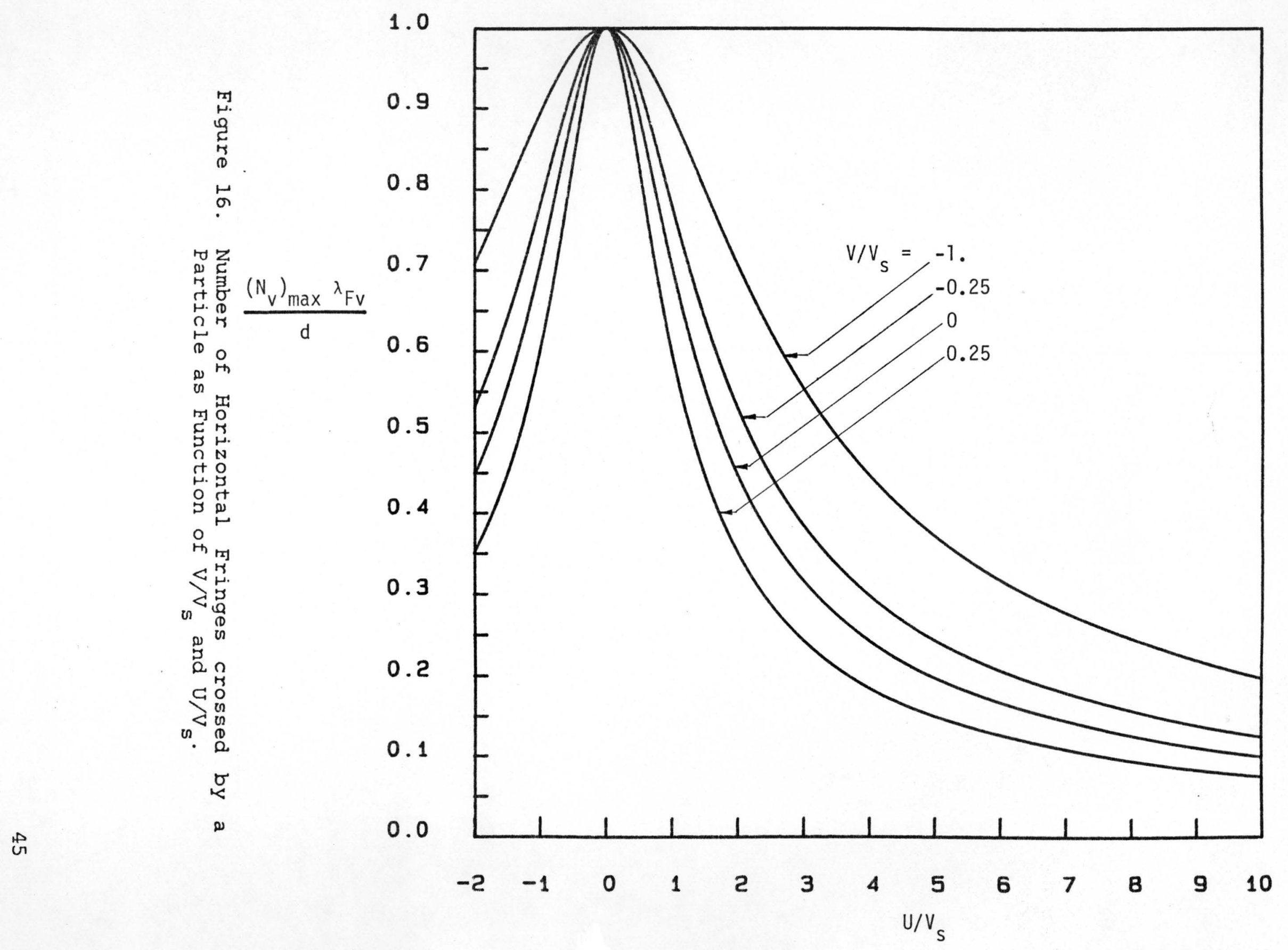


Figure $16 \mathrm{~b}$ shows the maximum of $\lambda_{\mathrm{FV}} \mathrm{N}_{\mathrm{V}} / \mathrm{d}$ plotted vesus $\mathrm{U} / \mathrm{V}_{\mathrm{S}}$ for different values of $\mathrm{V} / \mathrm{V}_{\mathrm{s}}$ ratio.

Using a counter which needs 32 cycles per signal burst, we can calculate the necessary horizontal fringe spacing $\lambda_{F V}$. For example, with a maximum streamwise velocity of $17 \mathrm{~m} / \mathrm{s}$, a measurement volume diameter of $3 / 4 \mathrm{~mm}$ viewed by the slit and a scan speed of $60 \mathrm{~m} / \mathrm{s}$, we get from eqn. (3), assuming $\left|\mathrm{V} / \mathrm{V}_{\mathrm{s}}\right|$ $<1:$

$$
\lambda_{E V}=22.5 \mu \mathrm{m}
$$

Velocity bias occurs because, according to the analysis of McLaughlin and Tiederman [5], the data rate is proportional to the magnitude of the instantaneous velocity of the fluid. Therefore, the velocity amplitude probability distribution is biased towards higher velocities. This biasing error, however, decreases using a scanning LDA in comparison with a pointwise LDA, considering the fact that the mean relative velocity of the fluid with respect to the measurement volume (scanning) is always greater than the mean velocity of the fluid (pointwise). It is also interesting to note that the bias decreases as the scan velocity is increased. 


\subsection{EXPERIMENTAL RESULTS}

V-component measurements were made using the Ronchi Ruling optics and the scanner. Two different types of measurements are performed:

- on a stationary solid object, consisting of a thin horizontal wire;

- in a dispersion of neutrally buoyant particles in water. Since the size of the wire and the particles influences the signal greatly, an appropriate fringe spacing needs to be chosen. An increase in particle size does not necessarily yield an increase in SNR as might be expected from the increase in light intensity with particle diameter. The modulation of the Doppler signal depends on the particle size and on the fringe spacing as is illustrated in Fig.17. A particle Pl with a diameter equivalent to one fringe spacing will always accomodate one half-dark fringe and one halfbright fringe. Hence, a position change of $\mathrm{Pl}$ inside the measurement volume only rearranges the position of intensity distribution over the particle cross section, but does not vary the scattered light intensity. 

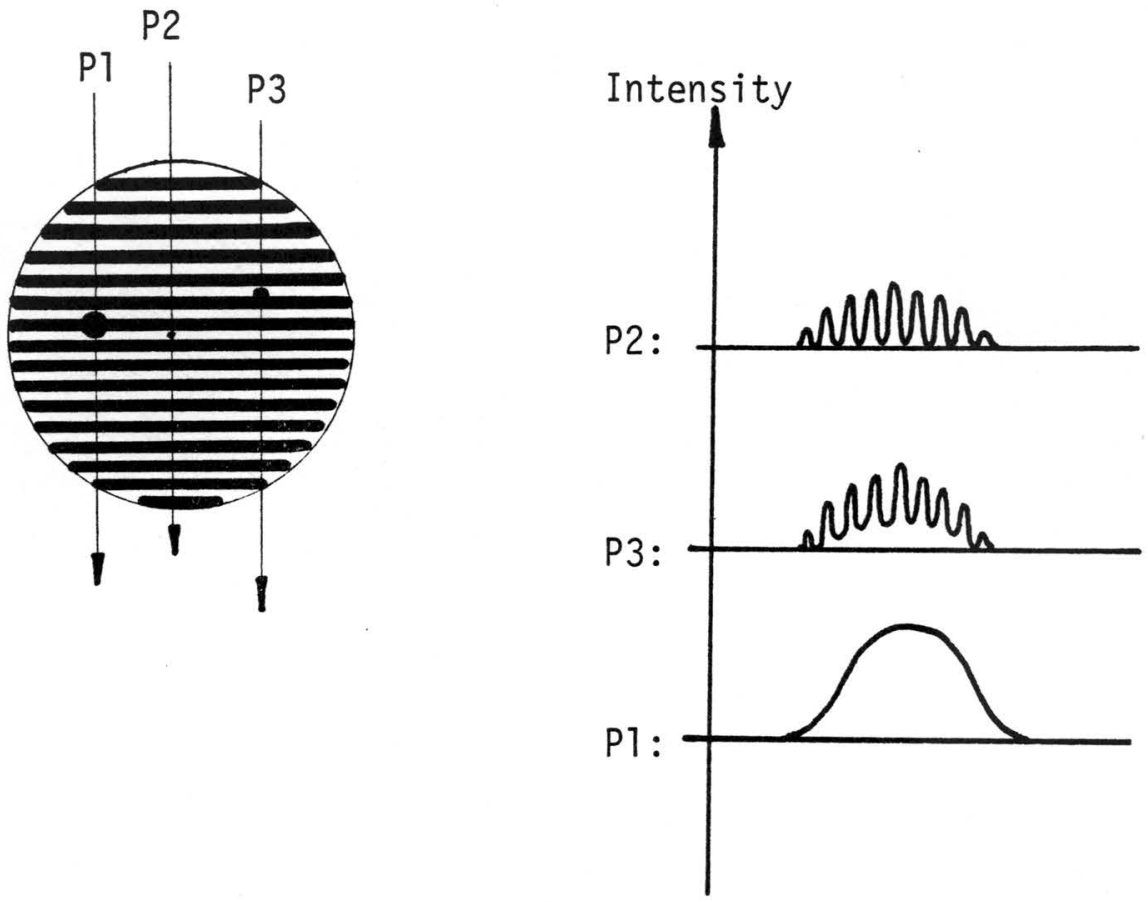

Size of $P 1=\lambda_{F}$

Size of $P 2 \leqslant \lambda_{F} / 2$

$\lambda_{F} / 2<$ Size of $\mathrm{P} 3<\lambda_{F} \quad\left(\lambda_{F}=\right.$ fringe spacing $)$

Figure 17. Influence of Particle Size on Doppler Signal Modulation. 


\subsubsection{MEASUREMENTS ON THIN STATIONARY WIRE}

A $25 \mu \mathrm{m}$ wire is installed horizontally as a light scatterer. Since it is stationary and it is being scanned in a vertical direction, the measured velocity $V$ should equal the scan velocity. The scan motion is generated by a sawtooth voltage to the scanner, hence, two different Doppler frequencies will be produced, one for the upward velocity $\left(V_{u}\right)$, and another for the downward velocity $\left(V_{d}\right)$. These frequencies are given by:

$$
\begin{aligned}
f_{u} & =v_{u} / \lambda_{F v} \\
& =\Delta y f_{s c a n} / \eta \lambda_{F V} \\
f_{d} & =\Delta y f_{s c a n} /(1-\eta) \lambda_{F v}
\end{aligned}
$$

where: $\lambda_{\mathrm{EV}} \quad=$ horizontal fringe spacing

$$
\begin{aligned}
\Delta y \quad= & \text { scan distance } \\
\eta \quad & \text { fraction of scan period } T \text {, corresponding } \\
& \text { to upward scan motion } \\
f_{\text {scan }}= & \text { scan frequency }
\end{aligned}
$$

Hence, for a scan distance of $40 \mathrm{~cm}$, scan frequency of $54 \mathrm{~Hz}$, with a $25 \mu \mathrm{m}$ horizontal fringe spacing, the upward velocity is :

$$
v_{u}=27.9 \mathrm{~m} / \mathrm{s},
$$

and the corresponding frequency: 


$$
f_{u}=1.00 \mathrm{MHz}
$$

However, since the wire diameter is $25 \mu \mathrm{m}$, a horizontal fringe spacing of $50 \mu \mathrm{m}$ was desired. This was obtained from the Ronchi Ruling by means of the transmitting lenses (L1,L2) as described earlier. The arrangement is shown in Fig.18. Doppler signals of very good quality were obtained with this configuration. Figure 19 shows the fringe visibility to be as high as $94 \%$. Very low noise levels are obtained.

\subsubsection{MEASUREMENTS IN DISPERSION OF NEUTRALLY BUOYANT PARTICLES IN WATER}

The same arrangement is used to make velocity measurements in water, using Polyaphrons as scattering particles. Added to water they will form a dispersion of individual Aphrons which are colored and have density equal to that of water. Using Aphrons of $25 \mu \mathrm{m}$ diameter and configuring the receiving optics in backscatter mode under an angle of $45^{\circ}$, the effective length of the measurement volume as seen by the PM-tube, becomes:

$$
\begin{aligned}
{ }_{\mathrm{MV}} & =d_{\mathrm{PM}} \mathrm{F}_{1} / \mathrm{F}_{2} \sin \phi \\
& =2.45 \mathrm{~mm}
\end{aligned}
$$




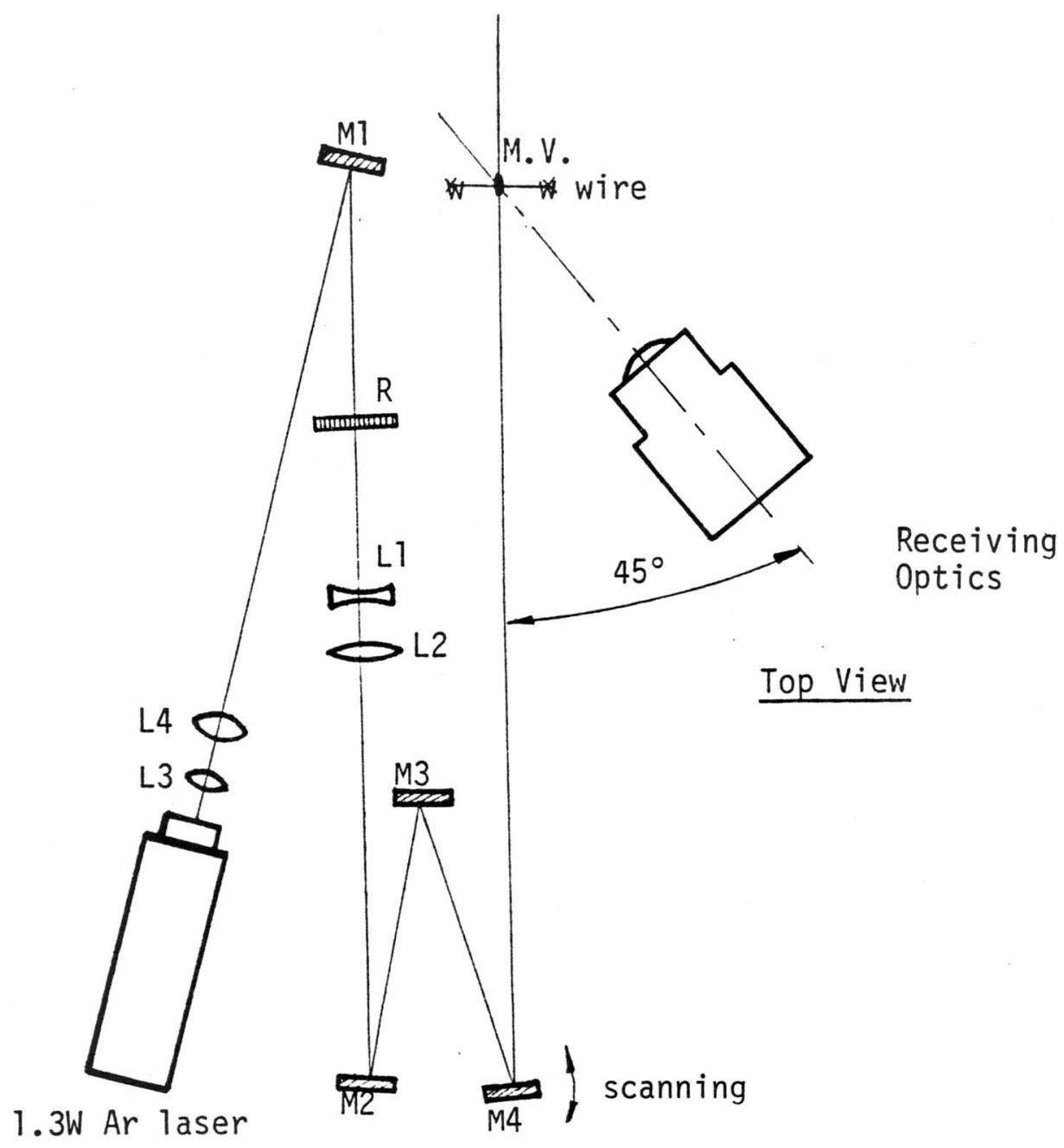

Figure 18. Arrangement for V-component Measurements. 
(a)

Horizontal Stationary Wire, Fringe Visibility $=94 \%$

(b)

Low noise level (detai1)

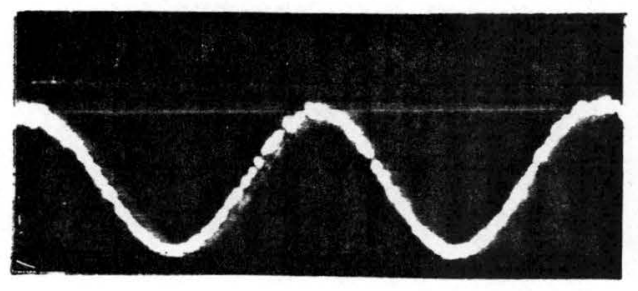

(c)

Moving Polyaphrons in water

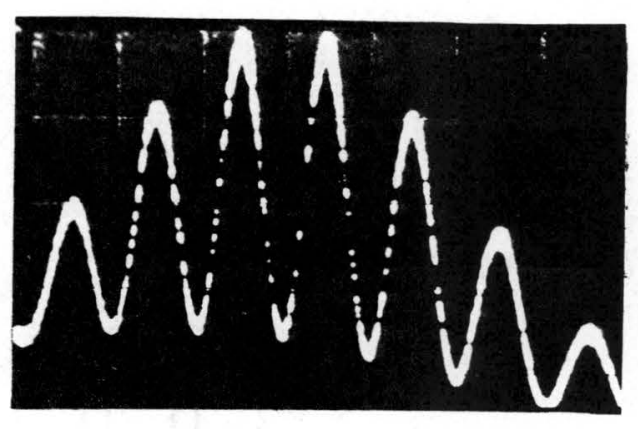

Figure 19. Doppler Signals for V-component. 
where: $\quad d_{P M}=$ PM-tube slit width $(0.5 \mathrm{~mm})$

$$
\begin{aligned}
& \mathrm{F}_{1}=300 \mathrm{~mm} \text { (cylindrical lens CL1) } \\
& \mathrm{F}_{2}=86 \mathrm{~mm} \text { (cylindrical lens CL2) }
\end{aligned}
$$

A Doppler signal of good quality was obtained with this configuration, as shown in Fig.19C. It was particularly modulated due to the high concentration of particles which led to the presence of more than one particle simultaneously present in the measurement volume. The SNR was proportional to the laser power in the measurement volume. It showed little dependence of the PM-tube slit width, although too small a slit attenuated the signal too much.

\section{Sources of Error}

An alignment error occured when the view of the PM-tube did not coincide with the measurement volume. Then light from different regions illuminated by the beams reached the detector. With low particle concentrations the misalignment is obvious since the production of a Doppler signal requires at least one particle in each of the regions simultaneously. In situations with high particle concentration however, a weak continuous signal was obtained. This was related to the velocities in the illuminated regions in the field of view 
and not to the velocity of a particle crossing the measurement volume.

Another source of error was stray light from reflections. This occured when measurements were made near a wall, and when the particle concentration was high. 
CHAPTER IV. FRINGE ANALYSIS FOR MEASURING U- AND W-

\section{COMPONENTS}

If there is a relative motion between interference fringes and the photodetector, the fringes are carried across the detector aperture and result in signals of variable light intensity. In a differential LDV system the two scattered light waves will interfere and yield a beat signal which has a frequency equal to the difference of the frequencies of the waves $\Delta v=v_{1}-v_{2}$. The main advantage of the differential LDV is that the frequency of the resulting signal is independent of the direction of light collection, and that there is no coherence limitation on the PM-tube slit width since the scattered waves that interfere are generated by one particle. Hence, large apertures can be utilized for the photodetector, resulting in higher SNR.

This optical beat phenomenon allows moving fringe patterns to be created. The fringes move with velocity $v_{f}$ given by :

$$
\begin{aligned}
v_{f} & =\lambda_{F} \Delta \nu \\
& =\lambda \Delta \nu /(2 \sin (\theta / 2))
\end{aligned}
$$

where: $\quad \lambda_{F}=$ fringe spacing

$$
\lambda=\text { wavelength of light }
$$




$$
\theta=\text { angle of intersection of the beams }
$$

The PM-tube sees moving fringes and gives an output signal at the beat frequency $\Delta \nu$.

\subsection{THE DUAL BRAGG CELL}

The most commonly used technique for directional discrimination involves frequency shifting of one or both of the crossing beams, which produces a moving fringe pattern. A particle moving in the same direction as the fringes will lower the signal frequency, whereas movement in the opposite direction will increase it.

In a Bragg cell acoustic waves through a transparent medium generate alternate layers of compression and expansion and, hence, change the index of refraction of that medium accordingly. If the angle of incidence of the incoming light wave is such that all reflections from the acoustic waves reinforce by interference, the resulting light beam is greatly enhanced. This angle is known as the Bragg angle. Diffracted beams are produced, but by orienting the cell to the Bragg angle most of the light can be concentrated in the first order of diffraction. If the index of refraction can be changed sinusoidally by the acoustic waves, at some frequency $f_{B}$ (Bragg frequency), then the outcoming light is phase modulated at $f_{B}$ (Debeye - Sears effect) (Eig.20). 
Hence, crossing such a beam with an unshifted beam produces moving a fringe pattern at frequency $f_{B^{\prime}}$ and the velocity of a particle $\left(v_{p}\right)$ in the measurement volume can then be obtained from:

$$
\begin{aligned}
v_{p} & =\lambda_{F}\left(f_{B}-f_{P}\right) \\
& =\lambda_{E} \cdot f_{D}
\end{aligned}
$$

where: $\quad \lambda_{E}=$ fringe spacing

$$
\begin{aligned}
& f_{p}=\text { frequency due to particle velocity } \\
& f_{D}=\text { measured Doppler frequency }
\end{aligned}
$$

The dual Bragg cell used in the present system has distilled water as medium for the acoustic waves, and employs transducers with nominal frequency of $5.042 \mathrm{MHz}$ and 8.036 $\mathrm{MHz}$. Their natural frequencies were measured to be $5.067 \mathrm{MHz}$ and $8.183 \mathrm{MHz}$ respectively. Driven at their $3 \mathrm{rd}$ overtone they produce Bragg frequencies of $15.200 \mathrm{MHz}$ and $24.550 \mathrm{MHz}$ for U- and W-velocity measurements. The driver unit of the Bragg cell transducers consists of Heathkit C.B. radio transmitters, Model DX-60 and DX-60B, using crystals of natural frequencies $7.600 \mathrm{MHz}$ and $8.183 \mathrm{MHz}$ amplified at their 2nd and 3 rd harmonic respectively. The output of the transmitters is matched to the input impedance of the transducers through impedance matching networks. 


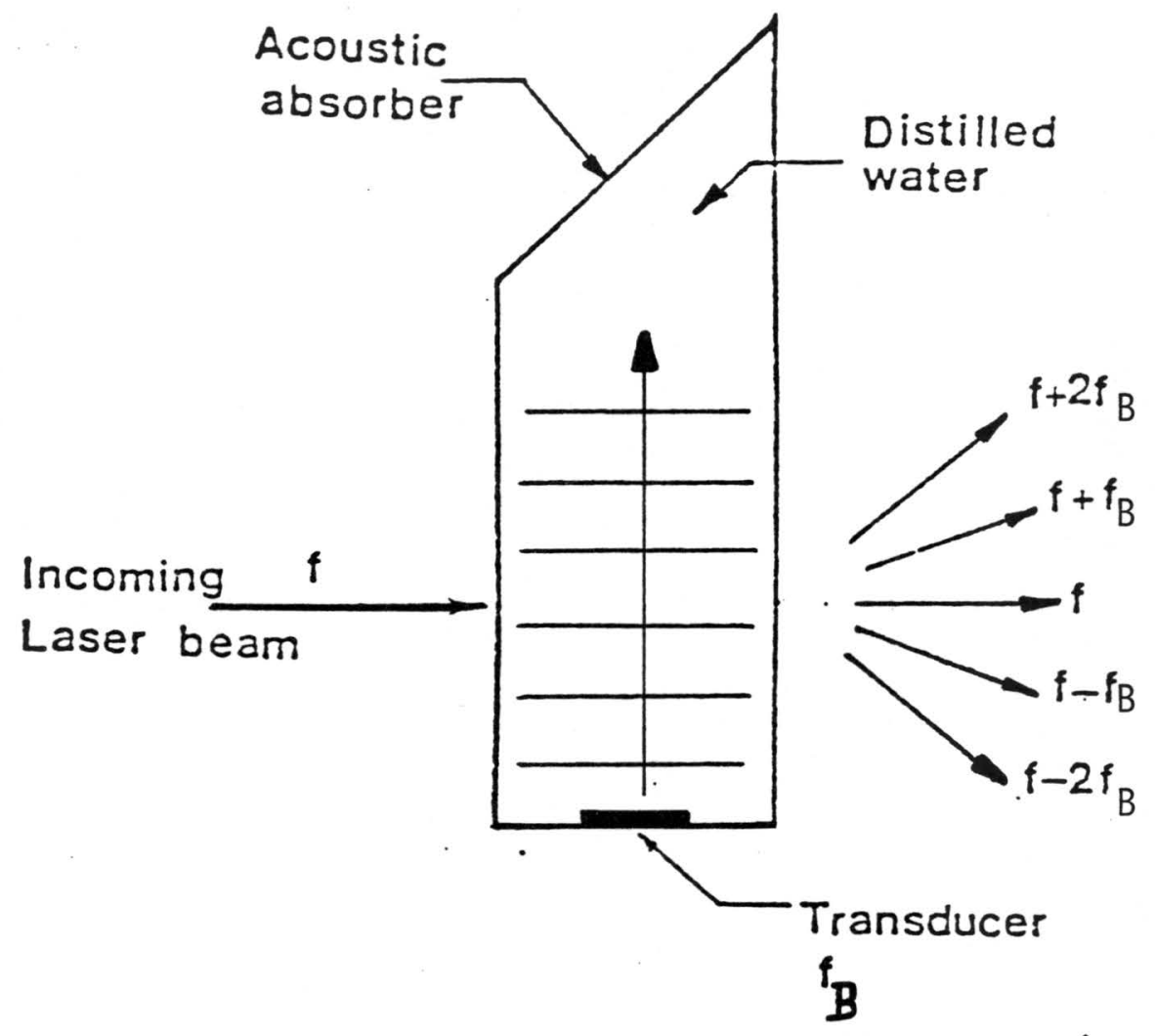

Figure 20. Frequency Shifting in Single Bragg cell. 


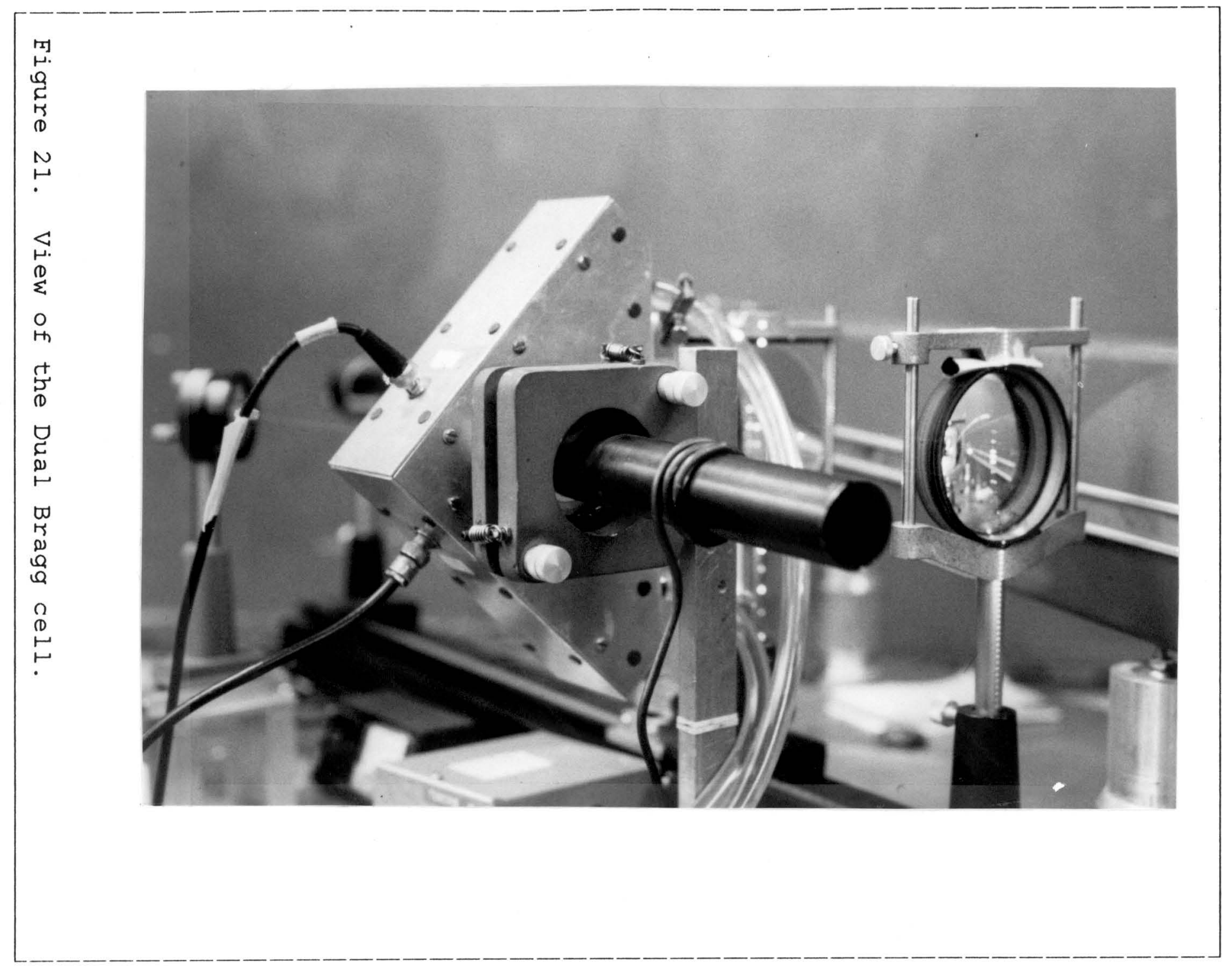


It can be shown that the angle of deviation, $\alpha$, of the outcoming shifted beams is given by:

$$
\sin \alpha / 2=\lambda / 2 \lambda_{A}
$$

where $\lambda$ is the optical wavelength and $\lambda_{A}$ is the acoustic wavelength in the medium [7]. A difficulty that can arise from the large difference between $\lambda$ and $\lambda_{A}$ is that the angle of deviation is small and separation of the diffracted beams is difficult. In our configuration however, Bragg frequencies of $15 \mathrm{MHz}$ and $25 \mathrm{MHz}$ are obtained with R.F. power of 1 to 2 Watt. At these frequencies and power level the diffraction angles were sufficient to allow easy separation.

\subsection{FRINGE PATTERNS FOR U- AND W-COMPONENTS}

We are interested in the intensity distribution resulting from the interference of three light waves of unequal frequencies.

Notation:

A plane light wave can be described as a real quantity $E$ as follows : 


$$
E=E_{0} \cos (2 \pi \nu t-\vec{k} \cdot \vec{r}+\phi)
$$

where: $E_{0}=$ amplitude of light wave

$$
\begin{array}{ll}
\nu & =\text { frequency } \\
\vec{k} & =\text { wave vector } \\
\vec{r} & =\text { position vector } \\
\phi & =\text { phase at origin at } t=0
\end{array}
$$

By introducing complex notation, $E$ can be written as the real part of an analytical signal $\varepsilon$ :

$$
E=R\{\varepsilon\}
$$

where: $\quad \varepsilon \equiv A \exp (i 2 \pi \nu t)$

and: $\quad A \equiv E_{0} \exp [-i(\vec{k} \cdot \vec{r}-\phi)]$

The light intensity is defined as the time average of the square of the electric field E :

$$
\begin{aligned}
I & \equiv 2 / T \int_{0}^{T} E^{2} d t \\
& =1 / 2 T \int_{0}^{T}\left(\varepsilon+\varepsilon^{\star}\right)^{2} d t
\end{aligned}
$$

(* denotes complex conjugate). 
The waves can be considered coherent, i.e. path length differences < coherence length of the source. Therefore the variations due to phase differences $\Delta \phi$ cannot be detected and, hence, we can ignore the phase $\phi$ in the expressions for the waves. Representing the three waves as in Fig.22,

i.e. $\quad \varepsilon_{i}=E_{0 i} \exp \left[j\left(\omega_{i} t-k \xi_{j}\right)\right]=A_{i} e^{j \omega_{j} t}$

$$
\text { where: } \begin{aligned}
\omega & \equiv 2 \pi \nu \\
k & =2 \pi / \lambda,
\end{aligned}
$$

the resulting fringe patterns can be found by superposition:

$$
\begin{aligned}
I & =1 / 2 T \int_{0}^{T}\left(\varepsilon_{1}+\varepsilon_{1}^{\star}+\varepsilon_{2}+\varepsilon_{2}^{*}+\varepsilon_{3}+\varepsilon_{3}^{\star}\right)^{2} d t \\
& =\sum_{n=1}^{3} A_{n} A_{n}^{\star}+1 / T \int_{0}^{T} \sum_{\substack{n=1 \\
n \neq m}}^{3}\left(\varepsilon_{n} \varepsilon_{m}+\varepsilon_{n} \varepsilon_{m}^{\star}+\varepsilon_{n}^{*} \varepsilon_{m}+\varepsilon_{n}^{\star} \varepsilon_{m}^{\star}\right)^{2} d t
\end{aligned}
$$

since: $\quad 1 / T \int_{0}^{T} \varepsilon^{2} d t=1 / T \int_{0}^{T} \varepsilon^{\star^{2}} d t=0$

and : $\quad 1 / T \int_{0}^{T} \varepsilon \varepsilon^{\star} d t=A A^{\star}$

$$
\text { Now let: }\left\{\begin{array}{l}
v_{1}=v \\
v_{2}=v+15 \mathrm{MHz} \\
v_{3}=v-25 \mathrm{MHz}
\end{array}\right.
$$




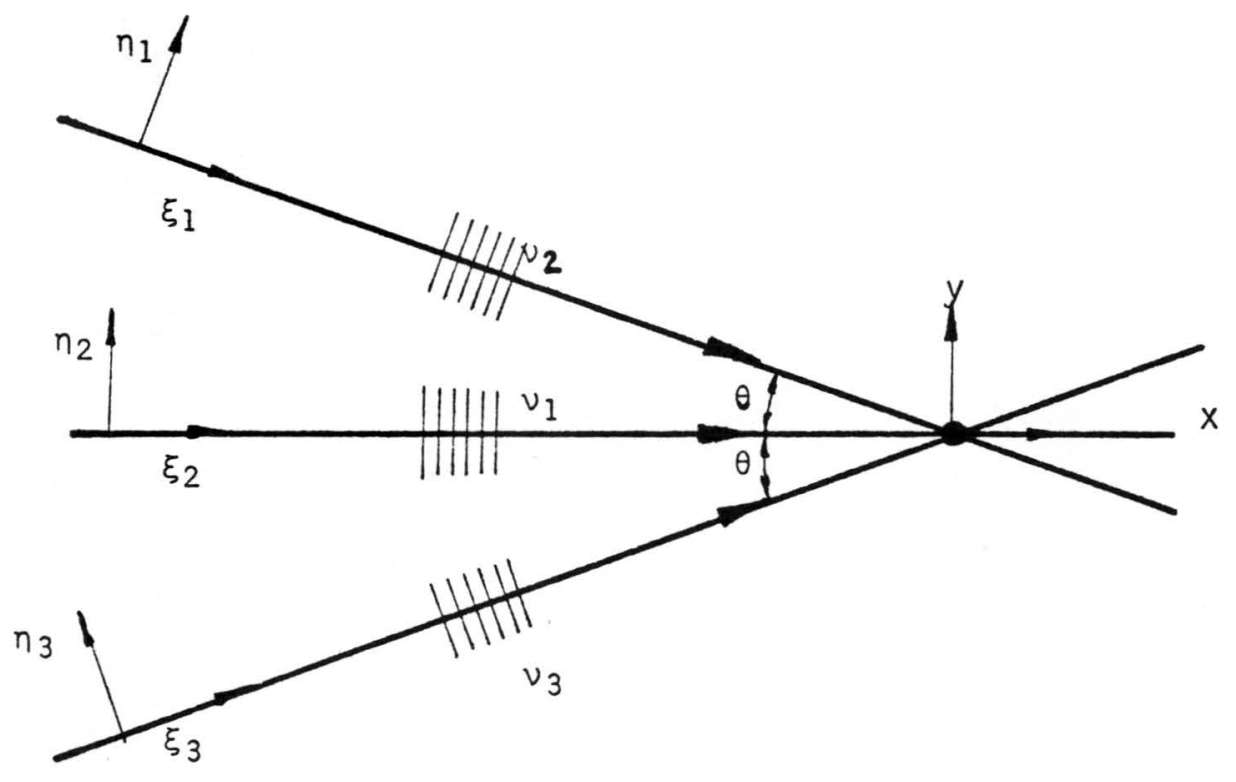

Eigure 22. Interference of Three Light Waves of Unequal Erequencies. 
Then the integral reduces to:

$$
\begin{aligned}
& \sum_{n=1}^{3} A_{n} A_{n}^{\star}+ \\
& 1 / T \int_{0}^{T} A_{1} A_{2}^{\star} \exp (-j 2 \pi 15 t)+A_{1} A_{3}^{\star} \exp (j 2 \pi 25 t)+A_{1} A_{2}^{\star} \exp (j 2 \pi 15 t) \\
& +A_{1} A_{3}^{*} \exp (-j 2 \pi 25 t)+A_{2} A_{3}^{*} \exp (j 2 \pi 40 t)+A_{2} A_{3}^{*} \exp (-j 2 \pi 40 t)
\end{aligned}
$$

since all other terms contain oscillations of order $v$, which integrate to zero because the frequency response of the PM-tube is too slow $(T>1 / v)$.

Now, since: $\quad A_{n} A_{m}^{*}=\left|E_{O n} E_{0 m}\right| \exp \left[j k\left(\xi_{n}-\xi_{m}\right)\right]$

the remaining terms can be written as:

$$
A_{n} A_{m}^{\star} e^{-j \omega t}+A_{n}^{*} A_{m} e^{j \omega t}=\left|E_{o n} E_{o m}\right| 2 \cos \left[k\left(\xi_{n}-\xi_{m}\right)+\omega t\right]
$$

and the resulting intensity distribution in the $(x, y)$-plane becomes:

$$
\begin{aligned}
& I(x, y, T)=C+ \\
& 2 / T \int_{0}^{T} \sum_{\substack{n=1,3 \\
n \neq m}}\left|E_{O n} E_{0 m}\right| \cos \left[k\left(\xi_{n}-\xi_{m}\right)+\left(\omega_{m}-\omega_{n}\right) t\right] d t
\end{aligned}
$$


where: $\quad C \equiv A_{1} A_{1}^{*}+A_{2} A_{2}^{*}+A_{3} A_{3}^{*}=$ const

$$
\begin{aligned}
& \xi_{1}=x \cos \theta-y \sin \theta \\
& \xi_{2}=x \\
& \xi_{3}=x \cos \theta+y \sin \theta
\end{aligned}
$$

The fringe pattern can then be found by calculating the minima and maxima of this intensity distribution.

The important conclusion, however, is that there is no intensity variation at $10 \mathrm{MHz}$. Therefore, a scheme relying on a $10 \mathrm{MHz}$ moving fringe pattern in the on-axis direction, as was proposed by TSI [8], cannot be successful. The only moving fringe patterns occur at $15 \mathrm{MHz}, 25 \mathrm{MHz}$ and $40 \mathrm{MHz}$ along off-axis directions.

These fringe patterns will permit the measurement of $U$ and $\mathrm{W}$, around frequencies of $10 \mathrm{MHz}$, and $40 \mathrm{MHz}$, as will be explained in the next section. 


\subsection{DOPPLER FREQUENCIES FOR U AND W}

The interference phenomena described above allow us to obtain Doppler signals around $\underline{40 \mathrm{MHz}}$ and $10 \mathrm{MHz}$ to measure U and $\mathrm{W}$ respectively, as will be shown.

A particle having three velocity components $\mathrm{U}, \mathrm{V}$ and $\mathrm{W}$ will yield five signals, due to the moving vertical fringes at 15 , 25, and $40 \mathrm{MHz}$ :

From Fig.23a,b:

$\lambda_{F W}\left(f_{1}-15\right)=-U \cos (\theta / 2) \cos \alpha-V \cos (\theta / 2) \sin \alpha-W \sin (\theta / 2)$

$\lambda_{F W}\left(f_{2}-15\right)=-U \cos (\theta / 2) \cos \alpha+V \cos (\theta / 2) \sin \alpha-W \sin (\theta / 2)$

$\lambda_{F W}\left(f_{3}-25\right)=-U \cos (\theta / 2) \cos \alpha+V \cos (\theta / 2) \sin \alpha+W \sin (\theta / 2)$

$\lambda_{E W}\left(f_{4}-25\right)=-U \cos (\theta / 2) \cos \alpha-V \cos (\theta / 2) \sin \alpha+W \sin (\theta / 2)$

From Fig. 23b:

$\lambda_{\mathrm{Fu}}\left(40-f_{5}\right)=U$

where: $\lambda_{F W}=\lambda / 2 \sin (\theta / 2)$

$\lambda_{\mathrm{Eu}}=\lambda / 2 \sin \theta$

$\theta \quad=$ half angle of intersection between frequencyshifted beams 

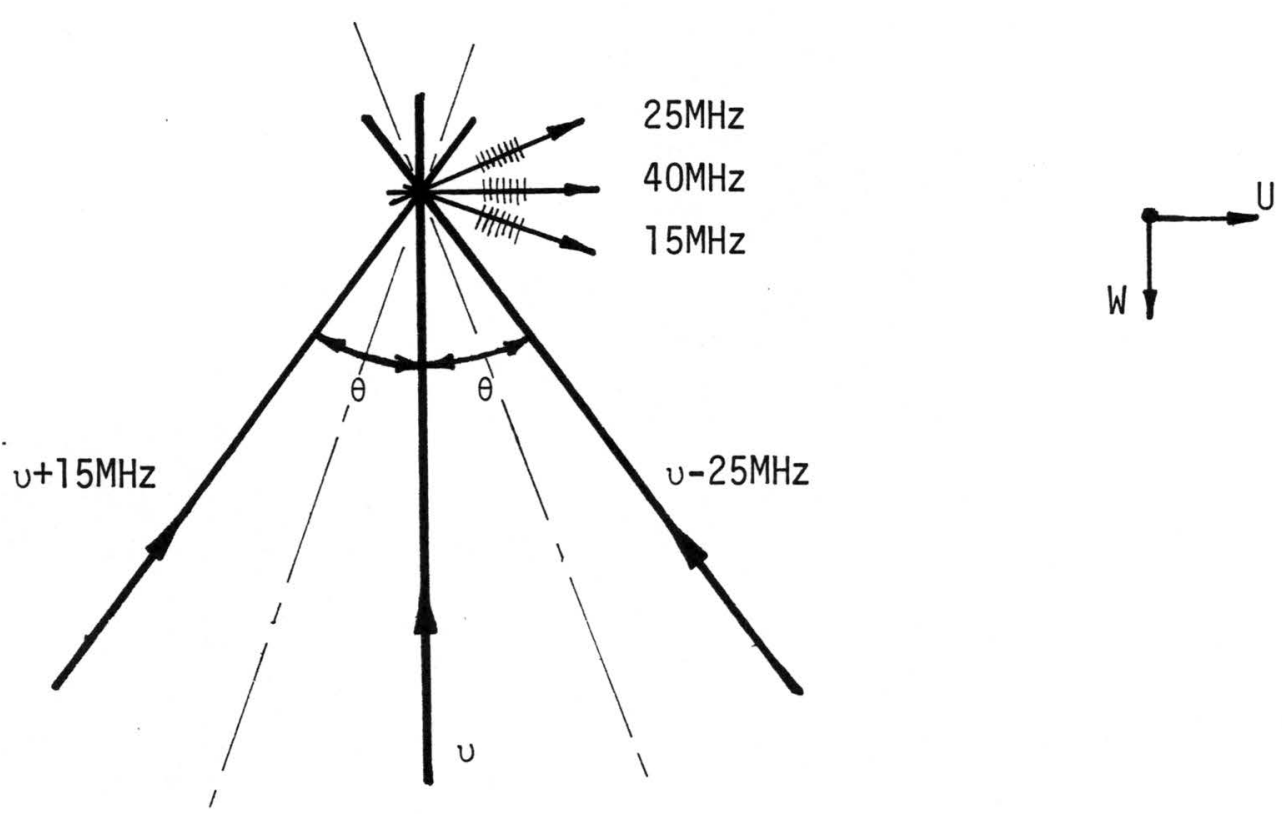

Top View

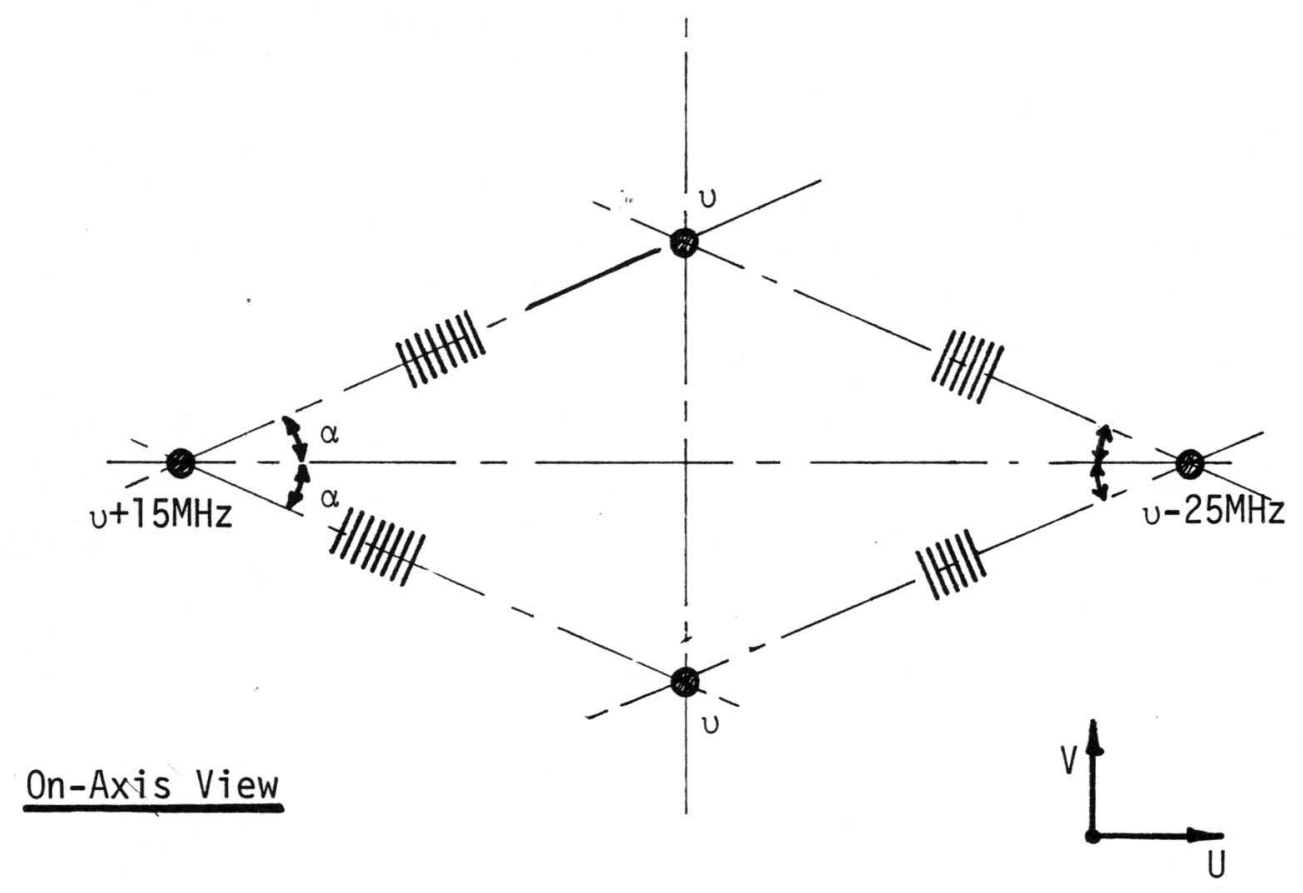

Figure 23. Fringe Patterns for $U$ and $W$. 


$$
f_{i}=\text { frequency due to particle motion }
$$

Hence, feeding the bandpass filtered signals around $15 \mathrm{MHz}$ and the bandpass filtered signals around $25 \mathrm{MHz}$ into a frequency mixer, yields $(1)+(2)-(3)-(4)$ :

$$
\lambda_{E W}\left(f_{1}+f_{2}-f_{3}-f_{4}-20\right)=-4 W \sin (\theta / 2)
$$

But, due to symmetry:

$$
f_{4}-f_{1}=f_{3}-f_{2}
$$

which gives an unambiguous way to measure $\mathrm{W}$ :

$$
W=\lambda_{E W}(f-10) / 2 \sin (\theta / 2)
$$

where: $\quad f=f_{3}-f_{2}$

$$
\begin{aligned}
= & f_{4}-f_{1} \\
= & \text { frequency resulting from mixing the bandpass } \\
& \text { filtered signals around } 15 \text { and } 25 \mathrm{MHz}
\end{aligned}
$$

Velocities in the $\mathrm{U}$ - and V-direction, however, cause the same increase in the $15 \mathrm{MHz}$ signal as they do for the $25 \mathrm{MHz}$ signal, and the difference between the two will always equal 10 $\mathrm{MHz}$. Therefore, signals around $40 \mathrm{MHz}$ will yield the Ucomponent, using equation (5). 


\subsection{EQUALIZING OPTICAL PATH LENGTHS}

The longitudinal (temporal) coherence of a light wave is primarily determined by the time-varying phase angle $\phi(t)$

Its influence on the fringe visibility is important. Considering the interference of two waves of equal frequency and ignoring the amplitude variations, it can easily be shown that the resulting intensity distribution is sinusoidal and contains a phase term $\Delta \phi(t)$, equal to the phase difference between the phases of the two waves. The optical path length (O.P.L.) difference influences the relative phase of the two waves at the screen, $\Delta \phi$, and, hence, determines the instantaneous position of the fringe pattern on the screen.

For nonzero, but small, path length difference, $\Delta \phi(t)$ remains small compared to $2 \pi$ and the fringe movement is small compared to the fringe spacing. The time-average fringe visibility is still high, the coherence near unity.

However, as the path length difference approaches the coherence length for the source the displacements become so great that the fringe pattern is 'washed out' and the timeaveraged fringe visibility goes to zero.

In the present optical configuration the difference in O.P.L. arises from the difference in paths travelled by the unshifted beams from the Ronchi Ruling and the shifted beams from the Bragg cell. However, by adjusting the position of mirror M4 only (Fig.1), the O.P.L. can be equalized. Hence, 
no additional path length equalizing optics are needed in this design.

\subsection{LASER POWER EFFICIENCY}

Different ratios can be defined and measured in order to monitor the laser power through the entire optical configuration.

For the Ronchi Ruling:

$$
\begin{aligned}
& n_{R 1} \equiv \frac{\text { output power }}{\text { input power }} \simeq 45 \% \\
& n_{R 2} \equiv \frac{\text { power of orders }-1,0,1}{\text { all diffracted power }} \simeq 70 \%
\end{aligned}
$$

For the Bragg cell:

$$
\begin{aligned}
& n_{B 1} \equiv \frac{\text { output power }}{\text { input power }} \simeq 93 \% \\
& n_{B 2} \equiv \frac{\text { power of } 15 \mathrm{MHz} \& 25 \mathrm{MHz}}{\text { input power in Bragg cell }} \simeq 44 \%
\end{aligned}
$$


Defining $\eta \equiv$ (Power in M.V.) / (Input Power), as an overall power ratio, this was found to equal, approximately, $22 \%$.

Although the Ronchi Ruling is rather inefficient due to reflection from its surface $\left(n_{R 1}\right)$, the loss in power due to the Bragg cell in this configuration is nearly as great. This is because the unshifted beam coming out of Bragg cell is not used in the measurement volume, but nevertheless contains a large fraction of the laser power. 
A rapidly scanning three-velocity-component laser Doppler anemometer was developed, to obtain space-time information in complicated 3-dimensional flows.

A horizontal fringe pattern for $\mathrm{V}$-component measurements was obtained with a Ronchi Ruling. Fringe visibility of $95 \%$ and low noise level signals were obtained on a solid object as well as in a fluid with scattering particles. For a scan frequency of $60 \mathrm{~Hz}$, a scan range of $40 \mathrm{~cm}$ and a fringe spacing of $25 \mu \mathrm{m}$, the Doppler frequency for $\mathrm{V}$ will be around $1 \mathrm{MHz}$.

Moving fringe patterns at 15,25 , and $40 \mathrm{MHz}$ were produced with a dual Bragg cell. Signals around $40 \mathrm{MHz}$ are a direct measurement of the U-velocity, and the difference of signals at 15 and $25 \mathrm{MHz}$ yields the W-velocity around 10 $\mathrm{MHz}$. It was proven that there was no moving fringe pattern in the on-axis direction at $10 \mathrm{MHz}$.

The optical configuration allows the size and location of the measurement volume, as well as all fringe spacings to be varied freely. Lens errors are reduced. No path length equalizing optics are necessary. The Doppler frequencies are independent of the position of the receiving optics, and only one PM-tube is needed. 
A disadvantage is the inefficient use of laser power and the requirements on mirror flatness and symmetry between the intersecting beams. 


\section{REFERENCES}

[1] Chehroudi, B., Simpson, R. L. 1983. Scanning LDA and its Applications in Turbulent Separated Flow, ONR Report SMU-WT-7.

[2] Chehroudi, B., Simpson, R. L. 1984. A Rapidly Scanning LDA, J. Phys. E: Sci. Instrum., Vol.16.

[3] Goodman, J. W. 1968. Introduction to Eourier Optics, McGraw-Hill.

[4] Hecht, E., Zajac, A. 1979. Optics, Addison Wesley.

[5] McLaughlin, D. K., Tiederman, W. G. 1973. Biasing Correction for Individual Realization of LDA Measurements in Flows, Physics of Fluids, Vol.16, Number 12, pp. 2082-2088.

[6] Durst, F., Melling, A., Whitelaw, J. H. 1976. Principles and Practice of Laser Doppler Anemometry, Academic Press.

[7] Drain, L. E. 1984. Laser Doppler Theory, John Wiley \& Sons. 
[8] TSI Inc., 1983. 3-Component, On-Axis LDV System, Technical Information.

[9] Bendick, P. J. 1971. A Laser Doppler Velocimeter to Measure Instantaneous Velocity Profiles, Proc. Flow Symp., May 10-14, Pittsburgh.

[10] Orloff, K. L., Biggers, J. C. 1974. Laser velocimeter Measurement of Developing and Periodic Flows, Proc. 2nd Int. Workshop on LDV, Purdue Vol.2, pp. 143-168.

[11] Orloff, K. L., Corsiglia, V. R., Biggers, J. C., Ekstedt, T. W. 1975. The Accuracy of Elow Measurements by Laser Doppler Methods, Proc. LDA Symp., Copenhagen, pp. 624-643.

[12] Rhodes, D. B. 1976. Optical Scanning System for Laser Velocimeter, SPIE 84, pp. 78-84.

[13] Gartrell, L. R., Jordan F. J. 1977. Demonstration of a Rapid-Scan Two-Dimensional Laser Velocimeter in the Langley Vortex Research Facility for Research 
in Aerial Applications, NASA Technical Note NASA TM-74081.

[14] Meyers, J. F., 1979. Application of Laser Velocimetry to Large Scale and Specialized Aerodynamic Tests, TSI Quart. 5 (4) 5-12.

[15] Nakatani, N., Yorisue, R., Yamada, T. 1978.

Simultaneous Measurements of Flow Velocities in Multipoint by the Laser Doppler Velocimeter, Proc. Dynamic Flow Conf., MarseilleBaltimore, pp. 583-590.

[16] Durst, F., Lehmann, B., Tropea, C. 1981. Laser Doppler System for Rapid Scanning of Flow Fields, Rev. Sci. Instrm. 52 1676-1681.

[17] Owen, F. K., 1984. A Scanning Laser Velocimeter for Turbulence Research, NASA Contractor Report 172493 . 
The vita has been removed from the scanned document 